\title{
Adaptive Harmonic Steady-State Control for Disturbance Rejection
}

\author{
Jaganath Chandrasekar, Li Liu, Dan Patt, Peretz P. Friedmann, and Dennis S. Bernstein
}

\begin{abstract}
We consider harmonic steady-state (HSS) control for active noise and vibration rejection when the system dynamics are unknown. After a brief review and analysis of the HSS control theory, we develop an adaptive control algorithm based on a recursive least squares algorithm that estimates the system dynamics. Active noise cancellation in an acoustic drum is demonstrated using the adaptive control algorithm. The results presented here unify and extend previous results on HSS control.
\end{abstract}

Index Terms-Acoustic applications, active noise control, adaptive control, disturbance rejection, harmonic steady state (HSS).

\section{NOMENCLATURE}

$\begin{array}{ll}\text { HSS } & \text { Harmonic steady state. } \\ \|\cdot\| & \text { Euclidean norm. } \\ X^{\mathrm{T}} & \text { Transpose of } X . \\ \operatorname{spec}(X) & \text { Spectrum of } X . \\ \operatorname{sprad}(X) & \text { Spectral radius of } X . \\ \sigma_{\max }(X) & \text { Largest singular value of } X . \\ I_{n} & n \times n \text { identity matrix. } \\ 0_{n} & n \times n \text { zero matrix. }\end{array}$

\section{INTRODUCTION}

$\mathbf{T}$ HE USE OF feedback control for disturbance rejection is of fundamental importance in a broad range of applications, and the development of effective algorithms is an ongoing area of research. For well-modelled plants with broadband disturbance, classical linear quadratic Gaussian (LQG) theory can be applied with weighting filters to shape the controller effort in accordance with the disturbance spectrum and performance objectives. For broadband disturbance rejection, there exists fundamental tradeoffs that imply that disturbance reduction over a given frequency range entails amplification at other frequencies [1].

On the other hand, if the disturbance is tonal or multitonal with known spectrum, then a model of the exogenous signal can be embedded in the controller to produce high-gain feedback

Manuscript received August 2, 2005; revised February 22, 2006. Manuscript received in final form May 19, 2006. Recommended by Associate Editor R. Rajamani. This work was supported in part by the Air Force Office of Scientific Research under Grant F429620-01-1-0094 and by the Army Research Office under Grant 02-1-0202.

The authors are with the Department of Aerospace Engineering, University of Michigan, Ann Arbor, MI 48108-2140 USA (e-mail: jchandra@engin.umich. edu; dsbaero@umich.edu).

Digital Object Identifier 10.1109/TCST.2006.880185 at frequencies that comprise the disturbance spectrum. The resulting internal model controller theoretically applies infinite gain at the disturbance frequencies to obtain asymptotically perfect disturbance rejection. The feedback signal can be realized equivalently by updating the coefficients of harmonic signals, which provides greater robustness in the event of sensor failure [2], [3]. In both implementations, guarantees of closed-loop stability require knowledge of the gain and phase of the plant at the disturbance frequencies, as well as additional modeling information.

When the plant and the disturbance are not well modeled, the problem can be significantly more challenging. Within the active noise control literature, numerous adaptive algorithms have been developed that were inspired by digital signal processing techniques. These techniques are based on LMS updating of finite-impulse response (FIR) filters (see [4]). These gradient-based algorithms often ignore the control-input-to-performance-variable secondary path transfer function [4], [5]. The FXLMS algorithm [6]-[8], on the other hand, is a variation of the LMS algorithm that takes into account the secondary path transfer function. Adaptive methods that estimate the secondary path transfer function have been used in conjunction with the FXLMS algorithm [9]. A delay in the secondary path transfer function results in a slow adaptation rate for the FXLMS algorithm, while uncertainty in the secondary path transfer function further reduces the convergence rate of the adaptive FXLMS algorithm [4, p. 128]. Modifications to the FXLMS algorithm to improve its convergence properties are developed in [10].

Another approach to disturbance rejection involves continuously adjusting the frequency, magnitude, and phase of the control input to cancel the disturbance [11], [12]. Alternative techniques, which require limited modeling of the plant dynamics and disturbance spectrum, have also been developed [13].

Yet another approach, which is applicable in the case of tonal or multitonal disturbances with known spectrum, allows the system to effectively reach harmonic steady-state (HSS) (that is, approximate sinusoidal response after transient decay) and uses measurements of the steady-state response amplitude and phase to determine the required amplitude and phase of the control signal. This technique was developed independently within two research communities. For helicopter vibration reduction, this technique is known as higher harmonic control, where the name reflects the aliasing effect of blade vibrations when transforming between the fixed and rotating reference frames [14]-[18]. The same technique was developed independently for active rotor balancing in this case, known as convergent control [19]. Helicopter vibration and rotor imbalance are, of course, closely related problems in which active disturbance 
rejection can be used to suppress the effect of harmonic disturbances with known spectrum due to load imbalance. We refer to this algorithm as HSS control. Connections between higher harmonic control and internal model control are discussed in [20].

Implementation of HSS control requires knowledge of the frequency response of the transfer function between the control input and the measurements at the disturbance frequency. In practice, this information is obtained through modeling or offline identification. When this information is uncertain or when the plant is subject to change, instability can occur. To address this issue, the robustness of HSS control is analyzed in [19] for additive and multiplicative model uncertainty. This analysis gives bounds for the modeling error that can be tolerated without instability. Of course, the level of disturbance rejection degrades when the modeled frequency response differs from the frequency response of the true system.

An adaptive extension of HSS control that removes the need to independently model the secondary path frequency response is considered in [21]. Specifically, a recursive least squares (RLS) (see [22]) procedure estimates this transfer function. In [23], a simultaneous estimation and control algorithm uses a moving-window batch (rather than recursive) least squares procedure to estimate the secondary path transfer function, where the estimation procedure is started only after a specified amount of control input and output data is obtained. Persistency of excitation in the estimation procedure is also addressed in [23].

In the present paper, we develop a unified framework for analyzing the properties and performance of nonadaptive and adaptive HSS control, extending much of the previous literature. We first analyze the robustness of nonadaptive HSS control and generalize the results of [19] to additive model uncertainty. In particular, we quantify the dependence of the performance and stability of nonadaptive HSS control on the estimate of the secondary path transfer function. To alleviate the need for offline modeling, we next consider an adaptive HSS control algorithm that uses the RLS procedure to estimate the secondary path transfer function and uses the estimate in the control update. This adaptive algorithm is direct with respect to the secondary path transfer function, since the identification is fully integrated with the control update.

The contents of the paper are as follows. In Sections II and IV, we review classical HSS control and demonstrate its onestep convergence property, a key feature that was apparently overlooked in the prior literature. Next, in Section V, we analyze the robustness and performance of nonadaptive HSS control. In Section VI, we discuss the use and implementation of a RLS algorithm for estimating the secondary path transfer function. Under suitable initialization, we show that the RLS estimate converges to the least squares solution. Convergence of the estimated transfer function and boundedness of the control signal are discussed in Section VII. In particular, we show that the performance of adaptive HSS control depends on the steady-state value of the RLS estimate of the transfer function. In Section VIII, we introduce adaptive HSS control with persistent excitation to ensure convergence of the RLS estimate to the actual secondary path transfer function. In Section VIIII,

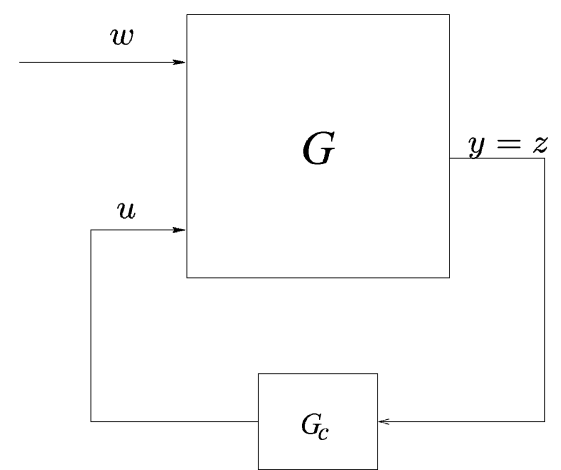

Fig. 1. Harmonic steady-state control architecture.

we numerically illustrate the adaptive HSS algorithm using an acoustic duct example involving two disturbance speakers, three actuation speakers, two microphones, and a dual-tone disturbance. This simulation is followed by an experimental demonstration involving active noise cancellation in an acoustic drum with one disturbance speaker, two actuation speakers, two microphones, and a single-tone disturbance. These examples show that an erroneous model of the system dynamics can cause instability in nonadaptive HSS control, whereas the adaptive HSS algorithm can recover stability. Some conclusions are given in Section XI.

\section{HARMONIC PERFORMANCE ANALYSIS}

Assume for convenience that the disturbance $w \in \mathbb{R}^{d}$ acting on the plant is a single harmonic with constant amplitude and phase. When the disturbance is a sum of sinusoids of multiple frequencies, the following analysis carries through with minor modifications. For details, see Appendix I. The HSS control algorithm waits until the output $y \in \mathbb{R}^{p}$ approximately reaches HSS, and then measures the amplitude and phase of the output. With this information, the control input $u \in \mathbb{R}^{m}$ is determined to minimize the effect of the disturbance $w$ on the output $y$. As shown in Fig. 1, the HSS control algorithm is a feedback controller, and thus, can potentially destabilize the plant, although not in the usual LTI sense. Note that the performance variable $z$ is the same as the output and, hence, $z=y$. We assume that the disturbance signal $w$ is unmeasured and, thus, is unavailable for feedback.

The inputs $u, w$, and the output $z$ are related by

$$
z=\left[\begin{array}{ll}
G_{z w} & G_{z u}
\end{array}\right]\left[\begin{array}{l}
w \\
u
\end{array}\right]
$$

where $G_{z w}(s)$ and $G_{z u}(s)$ are linear time-invariant multi-input multi-output (MIMO), continuous-time transfer functions with entries

$$
\begin{aligned}
& G_{z w}(s)=\left[\begin{array}{ccc}
G_{z_{1} w_{1}}(s) & \cdots & G_{z_{1} w_{d}}(s) \\
\vdots & & \vdots \\
G_{z_{p} w_{1}}(s) & \cdots & G_{z_{p} w_{d}}(s)
\end{array}\right] \\
& G_{z u}(s)=\left[\begin{array}{ccc}
G_{z_{1} u_{1}}(s) & \cdots & G_{z_{1} u_{m}}(s) \\
\vdots & & \vdots \\
G_{z_{p} u_{1}}(s) & \cdots & G_{z_{p} u_{m}}(s)
\end{array}\right] .
\end{aligned}
$$


In HSS control, the update of the control input $u$ is not performed continuously but rather at specified times $t_{k}$. The control input is harmonic with the same spectrum as the disturbance, and the amplitude and phase of the control input are updated at $t_{k}$. The time interval $t_{k+1}-t_{k}$ between two successive updates, need not be constant but must be sufficiently large to allow the output $z$ to effectively reach HSS. Measurement of the output $z$ is performed once $z$ has effectively reached HSS at which time $z$ is approximately harmonic with the same spectrum as the disturbance. Furthermore, the amplitude and phase of the output $z$ are completely determined by the amplitude and phase of the disturbance and control input. Assuming that the disturbance $w$ is harmonic with frequency $\omega_{1}$ and the output $z$ has approximately reached HSS within the time interval $\left[t_{k}, t_{k+1}\right]$, the signals $w(t), z(t)$, and $u(t)$ have the components

$$
\begin{aligned}
& w(t)=\left[\hat{w}_{1} \operatorname{Re}\left(e^{\jmath\left(\omega_{1} t+\phi_{1}\right)}\right) \cdots \hat{w}_{d} \operatorname{Re}\left(e^{\jmath\left(\omega_{1} t+\phi_{d}\right)}\right)\right]^{\mathrm{T}} \\
& z(t)=\left[\hat{z}_{1, k} \operatorname{Re}\left(e^{\jmath\left(\omega_{1} t+\theta_{1, k}\right)}\right) \cdots \hat{z}_{p, k} \operatorname{Re}\left(e^{\jmath\left(\omega_{1} t+\theta_{p, k}\right)}\right)\right]^{\mathrm{T}} \\
& u(t)=\left[\hat{u}_{1, k} \operatorname{Re}\left(e^{\jmath\left(\omega_{1} t+\psi_{1, k}\right)}\right) \cdots \hat{u}_{m, k} \operatorname{Re}\left(e^{\jmath\left(\omega_{1} t+\psi_{m, k}\right)}\right)\right]^{\mathrm{T}}
\end{aligned}
$$

where $\hat{w}_{i} \in \mathbb{R}, \hat{z}_{i, k} \in \mathbb{R}, \hat{u}_{i, k} \in \mathbb{R}$ are the amplitudes, and $\phi_{i} \in \mathbb{R}, \theta_{i, k} \in \mathbb{R}, \psi_{i, k} \in \mathbb{R}$ are the phase angles of the $i$ th components of $w(t), z(t)$, and $u(t)$, respectively.

Note that the amplitude $\hat{w}_{i}$ and phase $\phi_{i}$ of the $i$ th component of $w(t)$ are independent of the time interval $\left[t_{k}, t_{k+1}\right]$, and furthermore, $\phi_{i}$ is determined by the choice of $t_{0}$. The subscript $k$ in (2.5) and (2.6) indicates that the amplitude and phase of the components of $z(t)$ and $u(t)$ may be different in different time intervals.

For all $j=1, \ldots, p$, it follows from (2.1) that in HSS

$$
\begin{aligned}
\hat{z}_{j, k} \operatorname{Re}\left(e^{\jmath\left(\omega_{1} t+\theta_{j, k}\right)}\right)= & \sum_{i=1}^{d} \hat{w}_{i} \operatorname{Re}\left(G_{z_{j} w_{i}}\left(\jmath \omega_{1}\right) e^{\jmath\left(\omega_{1} t+\phi_{i}\right)}\right) \\
& +\sum_{i=1}^{m} \hat{u}_{i, k} \operatorname{Re}\left(G_{z_{j} u_{i}}\left(\jmath \omega_{1}\right) e^{\jmath\left(\omega_{1} t+\psi_{i, k}\right)}\right) .
\end{aligned}
$$

Note that, for all $i=1, \ldots, d$, where $\beta_{j i} \triangleq \angle G_{z_{j} u_{i}}\left(\jmath \omega_{1}\right)$.

Next, define $w_{\mathrm{s}_{i}}, w_{\mathrm{c}_{i}}, z_{\mathrm{s}_{i, k}}, z_{\mathrm{c}_{i, k}}, u_{\mathrm{s}_{i, k}}, u_{\mathrm{c}_{i, k}} \in \mathbb{R}$ by

$$
\begin{array}{rlrl}
w_{\mathrm{s}_{i}} & \triangleq-\hat{w}_{i} \sin \left(\phi_{i}\right), & w_{\mathrm{c}_{i}} \triangleq \hat{w}_{i} \cos \left(\phi_{i}\right) \\
z_{\mathrm{s}_{i, k}} \triangleq-\hat{z}_{i, k} \sin \left(\theta_{i, k}\right), & z_{\mathrm{c}_{i, k}} \triangleq \hat{z}_{i, k} \cos \left(\theta_{i, k}\right) \\
u_{\mathrm{s}_{i, k}} \triangleq-\hat{u}_{i, k} \sin \left(\psi_{i, k}\right), & u_{\mathrm{c}_{i, k}} \triangleq \hat{u}_{i, k} \cos \left(\psi_{i, k}\right) .
\end{array}
$$

Note that, for all $i=1, \ldots, d, w_{\mathrm{s}_{i}}$, and $w_{\mathrm{c}_{i}}$ are constants determined by the choice of $t_{0}$, and that $u_{\mathrm{s}_{i, k}}$ and $u_{\mathrm{c}_{i, k}}$ are determined by the control law. It then follows from (2.7)-(2.8) that, for all $j=1, \ldots, p$

$$
\left[\begin{array}{l}
z_{\mathrm{s}_{j, k}} \\
z_{\mathrm{c}_{j, k}}
\end{array}\right]=\sum_{i=1}^{d} W_{j i}\left[\begin{array}{l}
w_{\mathrm{s}_{i}} \\
w_{\mathrm{c}_{i}}
\end{array}\right]+\sum_{i=1}^{m} T_{j i}\left[\begin{array}{l}
u_{\mathrm{s}_{i, k}} \\
u_{\mathrm{c}_{i, k}}
\end{array}\right]
$$

where

$$
\begin{aligned}
T_{j i} & \triangleq\left[\begin{array}{cc}
\operatorname{Re}\left(G_{z_{j} w_{i}}\left(\jmath \omega_{1}\right)\right) & -\operatorname{Im}\left(G_{z_{j} w_{i}}\left(\jmath \omega_{1}\right)\right) \\
\operatorname{Im}\left(G_{z_{j} w_{i}}\left(\jmath \omega_{1}\right)\right) & \operatorname{Re}\left(G_{z_{j} w_{i}}\left(\jmath \omega_{1}\right)\right)
\end{array}\right] \\
W_{j i} & \triangleq\left[\begin{array}{cc}
\operatorname{Re}\left(G_{z_{j} u_{i}}\left(\jmath \omega_{1}\right)\right) & -\operatorname{Im}\left(G_{z_{j} u_{i}}\left(\jmath \omega_{1}\right)\right) \\
\operatorname{Im}\left(G_{z_{j} u_{i}}\left(\jmath \omega_{1}\right)\right) & \operatorname{Re}\left(G_{z_{j} u_{i}}\left(\jmath \omega_{1}\right)\right)
\end{array}\right] .
\end{aligned}
$$

Define $\bar{w} \in \mathbb{R}^{2 d}, \bar{z}_{k} \in \mathbb{R}^{2 p}$, and $\bar{u}_{k} \in \mathbb{R}^{2 m}$ by

$$
\begin{aligned}
& \bar{w} \triangleq\left[w_{\mathrm{s}_{1}} w_{\mathrm{c}_{1}} \cdots w_{\mathrm{s}_{d}} w_{\mathrm{c}_{d}}\right]^{\mathrm{T}} \\
& \bar{z}_{k} \triangleq\left[z_{\mathrm{s}_{1, k}} z_{\mathrm{c}_{1, k}} \cdots z_{\mathrm{s}_{p, k}} z_{\mathrm{c}_{p, k}}\right]^{\mathrm{T}} \\
& \bar{u}_{k} \triangleq\left[u_{\mathrm{s}_{1, k}} u_{\mathrm{c}_{1, k}} \cdots u_{\mathrm{s}_{m, k}} u_{\mathrm{c}_{m, k}}\right]^{\mathrm{T}} .
\end{aligned}
$$

It follows from (2.9) and (2.12) that the system dynamics in terms of $\bar{w}, \bar{z}_{k}$, and $\bar{u}_{k}$ are given by

$$
\bar{z}_{k}=T \bar{u}_{k}+W \bar{w}
$$

where $T \in \mathbb{R}^{2 p \times 2 m}$ and $W \in \mathbb{R}^{2 p \times 2 d}$ are defined by

$$
\begin{gathered}
T \triangleq\left[\begin{array}{ccc}
T_{11} & \cdots & T_{1 m} \\
\vdots & & \vdots \\
T_{p 1} & \cdots & T_{p m}
\end{array}\right] \\
W \triangleq\left[\begin{array}{ccc}
W_{11} & \cdots & W_{1 d} \\
\vdots & & \vdots \\
W_{p 1} & \cdots & W_{p d}
\end{array}\right] .
\end{gathered}
$$

Replacing $k$ by $k+1$ in (2.13) and subtracting the resulting equation from (2.13), yields the disturbance-free update model

$$
\bar{z}_{k+1}=\bar{z}_{k}+T\left(\bar{u}_{k+1}-\bar{u}_{k}\right) .
$$

\section{HSS ALGORITHM}

Consider the cost function

$$
J\left(\bar{z}_{k}, \bar{u}_{k}\right) \triangleq \bar{z}_{k}^{\mathrm{T}} Q \bar{z}_{k}+2 \bar{z}_{k}^{\mathrm{T}} S \bar{u}_{k}+\bar{u}_{k}^{\mathrm{T}} R \bar{u}_{k}
$$

where $Q \in \mathbb{R}^{2 p \times 2 p}, S \in \mathbb{R}^{2 p \times 2 m}$, and $R \in \mathbb{R}^{2 m \times 2 m}$ are weighting matrices such that $\left[\begin{array}{cc}Q & S \\ S^{\mathrm{T}} & R\end{array}\right]$ is positive definite. Substituting $\bar{z}_{k}$ from (2.13) into (3.1) yields

$$
J\left(\bar{z}_{k}, \bar{u}_{k}\right)=\bar{u}_{k}^{\mathrm{T}} D \bar{u}_{k}+2 \bar{u}_{k}^{\mathrm{T}}\left(T^{\mathrm{T}} Q+S^{\mathrm{T}}\right) W \bar{w}+\bar{w}^{\mathrm{T}} \tilde{Q} \bar{w}
$$

where $\tilde{Q} \triangleq W^{\mathrm{T}} Q W$ and the positive-definite matrix $D$ is defined by

$$
D \triangleq T^{\mathrm{T}} Q T+T^{\mathrm{T}} S+S^{\mathrm{T}} T+R .
$$

Since (3.2) involves only $\bar{u}_{k}$ and $\bar{w}$, we define

$$
\mathcal{J}\left(\bar{w}, \bar{u}_{k}\right) \triangleq J\left(\bar{z}_{k}, \bar{u}_{k}\right)
$$

and write $\mathcal{J}\left(\bar{w}, \bar{u}_{k}\right)$ as

$\mathcal{J}\left(\bar{w}, \bar{u}_{k}\right)=\left[(W \bar{w})^{\mathrm{T}} \bar{u}_{k}^{\mathrm{T}}\right]\left[\begin{array}{cc}Q & Q T+S \\ T^{\mathrm{T}} Q+S^{\mathrm{T}} & D\end{array}\right]\left[\begin{array}{c}W \bar{w} \\ \bar{u}_{k}\end{array}\right]$. 
To determine $\bar{u}_{k}$ that minimizes $\mathcal{J}\left(\bar{w}, \bar{u}_{k}\right)$, we set

$$
\frac{\partial \mathcal{J}\left(\bar{w}, \bar{u}_{k}\right)}{\partial \bar{u}_{k}}=2 D \bar{u}_{k}+2\left(T^{\mathrm{T}} Q+S^{\mathrm{T}}\right) W \bar{w}=0 .
$$

The optimal control law is given by

$$
\bar{u}_{\mathrm{opt}} \triangleq \bar{u}_{k, \mathrm{opt}}=-D^{-1}\left(T^{\mathrm{T}} Q+S^{\mathrm{T}}\right) W \bar{w}
$$

and the minimum cost is

$$
\mathcal{J}\left(\bar{w}, \bar{u}_{\text {opt }}\right)=(W \bar{w})^{\mathrm{T}}\left[Q-M^{\mathrm{T}} D M\right] W \bar{w}
$$

where $M \in \mathbb{R}^{2 m \times 2 p}$ is defined by

$$
M \triangleq D^{-1}\left(T^{\mathrm{T}} Q+S^{\mathrm{T}}\right) .
$$

Since $\bar{u}_{\text {opt }}$ depends on $\bar{w}$, whose measurement is not available, we derive an equivalent control law that can be used for all $k \geqslant$ 1.

Setting $k=0$ in (2.13), yields

$$
W \bar{w}=\bar{z}_{0}-T \bar{u}_{0}
$$

and, hence, substituting (3.10) into (2.13) yields

$$
\bar{z}_{k}=\bar{z}_{0}+T\left(\bar{u}_{k}-\bar{u}_{0}\right) .
$$

From (3.10) the optimal control law $\bar{u}_{\text {opt }}$ in (3.7) can be written as

$$
\bar{u}_{\mathrm{opt}}=-D^{-1}\left(T^{\mathrm{T}} Q+S^{\mathrm{T}}\right)\left(\bar{z}_{0}-T \bar{u}_{0}\right)
$$

and the minimum cost in (3.8) can be expressed as

$$
\mathcal{J}\left(\bar{w}, \bar{u}_{\text {opt }}\right)=\left(\bar{z}_{0}-T \bar{u}_{0}\right)^{\mathrm{T}}\left[Q-M^{\mathrm{T}} D M\right]\left(\bar{z}_{0}-T \bar{u}_{0}\right) .
$$

Finally, as in [17] and [18], assume that $S=0$ so that the optimal control law (3.12) is given by

$$
\bar{u}_{\mathrm{opt}}=-D^{-1} T^{\mathrm{T}} Q\left(\bar{z}_{0}-T \bar{u}_{0}\right)
$$

and the minimum cost (3.13) is

$\mathcal{J}\left(\bar{w}, \bar{u}_{\mathrm{opt}}\right)=\left(\bar{z}_{0}-T \bar{u}_{0}\right)^{\mathrm{T}}\left(Q-Q T D^{-1} T^{\mathrm{T}} Q\right)\left(\bar{z}_{0}-T \bar{u}_{0}\right)$.

\section{Convergence Analysis of the HSS Algorithm}

Note that $\bar{u}_{\text {opt }}$ given by (3.12) is independent of $k$, and hence, remains constant for all $k \geqslant 1$. Substituting (3.12) into (3.11) and (3.1), the optimal value of $\bar{z}_{k}$ for all $k \geqslant 1$ is given by

$$
\bar{z}_{\mathrm{opt}} \triangleq \bar{z}_{k, \mathrm{opt}}=\left[I-T D^{-1}\left(T^{\mathrm{T}} Q+S^{\mathrm{T}}\right)\right]\left(\bar{z}_{0}-T \bar{u}_{0}\right)
$$

and, thus

$$
J\left(\bar{z}_{\text {opt }}, \bar{u}_{\text {opt }}\right)=\left(\bar{z}_{0}-T \bar{u}_{0}\right)^{\mathrm{T}}\left[Q-M^{\mathrm{T}} D M\right]\left(\bar{z}_{0}-T \bar{u}_{0}\right) .
$$

Using (3.11) in (3.12), the optimal control law can be expressed recursively as

$$
\bar{u}_{k+1, \mathrm{opt}}=-D^{-1}\left(T^{\mathrm{T}} Q+S^{\mathrm{T}}\right)\left(\bar{z}_{k, \mathrm{opt}}-T \bar{u}_{k, \mathrm{opt}}\right) .
$$

The state-space representation of the system dynamics with the optimal control law is

$$
\left[\begin{array}{l}
\bar{z}_{k+1, \mathrm{opt}} \\
\bar{u}_{k+1, \mathrm{opt}}
\end{array}\right]=A\left[\begin{array}{l}
\bar{z}_{k, \mathrm{opt}} \\
\bar{u}_{k, \mathrm{opt}}
\end{array}\right]
$$

where $A \in \mathbb{R}^{2(p+m) \times 2(p+m)}$ is defined by

$$
A \triangleq\left[\begin{array}{cc}
I_{2 p}-T M & -\left(I_{2 p}-T M\right) T \\
-M & M T
\end{array}\right] .
$$

Next, note that

$$
A^{2}=\left[\begin{array}{cc}
I_{2 p}-T M & -\left(I_{2 p}-T M\right) T \\
-M & M T
\end{array}\right]=A .
$$

Hence, $A$ is an idempotent matrix, and its eigenvalues are either 0 or 1 . In fact, $A$ can be factored as

$$
A=\left[\begin{array}{cc}
I_{2 p} & T \\
0 & I_{2 m}
\end{array}\right]\left[\begin{array}{cc}
I_{2 p} & 0 \\
-M & 0_{2 m}
\end{array}\right]\left[\begin{array}{cc}
I_{2 p} & -T \\
0 & I_{2 m}
\end{array}\right]
$$

which shows that

$$
\operatorname{spec}(A)=\operatorname{spec}\left(I_{2 p}\right) \cup \operatorname{spec}\left(0_{2 m}\right) .
$$

With the initial conditions $\bar{z}_{0}$ and $\bar{u}_{0}$, (4.4) implies that

$$
\left[\begin{array}{l}
\bar{z}_{1, \mathrm{opt}} \\
\bar{u}_{1, \mathrm{opt}}
\end{array}\right]=A\left[\begin{array}{l}
\bar{z}_{0} \\
\bar{u}_{0}
\end{array}\right]
$$

and

$$
\left[\begin{array}{l}
\bar{z}_{2, \mathrm{opt}} \\
\bar{u}_{2, \mathrm{opt}}
\end{array}\right]=A^{2}\left[\begin{array}{l}
\bar{z}_{0} \\
\bar{u}_{0}
\end{array}\right]=A\left[\begin{array}{l}
\bar{z}_{0} \\
\bar{u}_{0}
\end{array}\right]=\left[\begin{array}{l}
\bar{z}_{1, \mathrm{opt}} \\
\bar{u}_{1, \mathrm{opt}}
\end{array}\right] .
$$

Similarly, for all $k=1,2, \ldots$,

$$
\begin{aligned}
{\left[\begin{array}{l}
\bar{z}_{\text {opt }} \\
\bar{u}_{\text {opt }}
\end{array}\right] } & =\left[\begin{array}{c}
\bar{z}_{k, \mathrm{opt}} \\
\bar{u}_{k, \mathrm{opt}}
\end{array}\right] \\
& =A\left[\begin{array}{l}
\bar{z}_{0} \\
\bar{u}_{0}
\end{array}\right]=\left[\begin{array}{c}
(I-T M)\left(\bar{z}_{0}-T \bar{u}_{0}\right) \\
-M\left(\bar{z}_{0}-T \bar{u}_{0}\right)
\end{array}\right] .
\end{aligned}
$$

Consequently, the optimal values of $\bar{u}_{\mathrm{opt}}$ in (3.12) and $\bar{z}_{\mathrm{opt}}$ in (4.1) are attained after the first update.

In the case $S=0, \bar{z}_{\mathrm{opt}}$ in (4.1), can be expressed as

$$
\begin{aligned}
\bar{z}_{\mathrm{opt}}= & {\left[I-T R^{-1 / 2}\left(I+R^{-1 / 2} T^{\mathrm{T}} Q T R^{-1 / 2}\right)^{-1}\right] } \\
& \times\left(\bar{z}_{0}-T \bar{u}_{0}\right) \\
= & Q^{-1}\left(Q^{-1}+T R^{-1} T^{\mathrm{T}}\right)^{-1}\left(\bar{z}_{0}-T \bar{u}_{0}\right) .
\end{aligned}
$$

Hence

$$
\left\|\bar{z}_{\mathrm{opt}}\right\| \leqslant \frac{\sigma_{\max }\left(\left(Q^{-1}+T R^{-1} T^{\mathrm{T}}\right)^{-1}\right)}{\sigma_{\min }(Q)}\left\|\bar{z}_{0}-T \bar{u}_{0}\right\| .
$$


Hence, if $2 m<2 p$ and $\sigma_{\min }(T)>0$, then

$$
\left\|\bar{z}_{\mathrm{opt}}\right\| \leqslant \frac{\sigma_{\max }(R)}{\sigma_{\min }(Q) \sigma_{\min }(T)^{2}}\left\|\bar{z}_{0}-T \bar{u}_{0}\right\| .
$$

If $\sigma_{\max }(R) / \sigma_{\min }(Q)$ is large (minimum energy control), it follows from (4.13) that $\left\|\bar{z}_{\text {opt }}\right\|$ may be large, indicating poor performance. Alternatively, if $\sigma_{\max }(R) / \sigma_{\min }(Q)$ is small (cheap control), then (4.13) implies that the $\left\|\bar{z}_{\text {opt }}\right\|$ is small, and hence, the performance is good.

\section{ROBUSTNESS OF HSS CONTROL}

Implementation of HSS control requires knowledge of $T$. An erroneous model of $T$ can result in degraded performance and possible instability. When an estimate $\hat{T}$ of $T$ is given, the control law defined in (4.3), becomes

$$
\hat{\bar{u}}_{k+1}=-\hat{M}\left(\bar{z}_{k}-\hat{T} \hat{\bar{u}}_{k}\right)
$$

where

$$
\begin{gathered}
\hat{M} \triangleq \hat{D}^{-1}\left(\hat{T}^{\mathrm{T}} Q+S^{\mathrm{T}}\right) \in \mathbb{R}^{2 m \times 2 p} \\
\hat{D} \triangleq \hat{T}^{\mathrm{T}} Q \hat{T}+S^{\mathrm{T}} \hat{T}+\hat{T}^{\mathrm{T}} S+R .
\end{gathered}
$$

The state-space representation of the system dynamics with (5.1) is

$$
\left[\begin{array}{l}
\bar{z}_{k+1} \\
\hat{\bar{u}}_{k+1}
\end{array}\right]=\hat{A}\left[\begin{array}{c}
\bar{z}_{k} \\
\overline{\bar{u}}_{k}
\end{array}\right]
$$

where $\hat{A}$ is defined by

$$
\hat{A} \triangleq\left[\begin{array}{cc}
I_{2 p}-T \hat{M} & T\left(\hat{M} \hat{T}-I_{2 p}\right) \\
-\hat{M} & \hat{M} \hat{T}
\end{array}\right]
$$

and $\Delta T \triangleq \hat{T}-T$.

It is useful to factor $\hat{A}$ as

$$
\hat{A}=\left[\begin{array}{cc}
I_{2 p} & T \\
0 & I_{2 m}
\end{array}\right]\left[\begin{array}{cc}
I_{2 p} & 0 \\
-\hat{M} & \hat{M} \Delta T
\end{array}\right]\left[\begin{array}{cc}
I_{2 p} & -T \\
0 & I_{2 m}
\end{array}\right]
$$

which shows that

$$
\operatorname{spec}(\hat{A})=\operatorname{spec}\left(I_{2 p}\right) \cup \operatorname{spec}(\hat{M} \Delta T) .
$$

Hence, the HSS algorithm is stable if and only if

$$
\operatorname{sprad}(\hat{M} \Delta T)<1 .
$$

In the special case $S=0$, it follows that

$$
\begin{aligned}
\operatorname{sprad}(\hat{M} \Delta T) & =\operatorname{sprad}\left(\left(\hat{T}^{\mathrm{T}} Q \hat{T}+R\right)^{-1} \hat{T}^{\mathrm{T}} Q \Delta T\right) \\
& \leqslant \sigma_{\max }\left(\left(\hat{T}^{\mathrm{T}} Q \hat{T}+R\right)^{-1} \hat{T}^{\mathrm{T}} Q \Delta T\right) \\
& \leqslant \frac{\sigma_{\max }(T+\Delta T) \sigma_{\max }(Q) \sigma_{\max }(\Delta T)}{\sigma_{\min }(R)} .
\end{aligned}
$$

Therefore, if

$$
\sigma_{\max }(\Delta T)<\frac{-\sigma_{\max }(T)}{2}+\frac{1}{2} \sqrt{\sigma_{\max }(T)^{2}+4 \frac{\sigma_{\min }(R)}{\sigma_{\max }(Q)}}
$$

then $\operatorname{sprad}(\hat{M} \Delta T)<1$. If $\sigma_{\min }(R) / \sigma_{\max }(Q)$ is large (minimum energy control), then according to (5.9), HSS control possesses a high degree of robustness. However, if $\sigma_{\min }(R) / \sigma_{\max }(Q)$ is small (cheap control), then (5.9) implies that robustness is compromised.

From (5.5), it follows that

$$
\hat{A}^{k}=\left[\begin{array}{cc}
I_{2 p} & T \\
0 & I_{2 m}
\end{array}\right]\left[\begin{array}{cc}
I_{2 p} & 0 \\
\hat{A}_{21, k} & (\hat{M} \Delta T)^{k}
\end{array}\right]\left[\begin{array}{cc}
I_{2 p} & -T \\
0 & I_{2 m}
\end{array}\right]
$$

where $\hat{A}_{21, k} \triangleq-\sum_{i=1}^{k-1}(\hat{M} \Delta T)^{i} \hat{M}$. Now assume that HSS control is stable, that is, (5.7) is satisfied. In this case

$$
\lim _{k \rightarrow \infty}(\hat{M} \Delta T)^{k}=0
$$

and

$$
\Gamma \triangleq \lim _{k \rightarrow \infty} \sum_{i=1}^{k-1}(\hat{M} \Delta T)^{i}=(I-\hat{M} \Delta T)^{-1} .
$$

Hence, (5.10), (5.11), and (5.12) imply that

$$
\lim _{k \rightarrow \infty} \hat{A}^{k}=\left[\begin{array}{cc}
I-T \Gamma \hat{M} & T(\Gamma \hat{M} T-I) \\
-\Gamma \hat{M} & \Gamma \hat{M} T
\end{array}\right] .
$$

The limiting values of $\bar{z}_{k}$ and $\hat{\bar{u}}_{k}$ are given by

$$
\begin{aligned}
\lim _{k \rightarrow \infty}\left[\begin{array}{c}
\bar{z}_{k} \\
\hat{\bar{u}}_{k}
\end{array}\right] & =\lim _{k \rightarrow \infty} \hat{A}^{k}\left[\begin{array}{c}
\bar{z}_{0} \\
\hat{\bar{u}}_{0}
\end{array}\right] \\
& =\left[\begin{array}{c}
(I-T \Gamma \hat{M})\left(\bar{z}_{0}-T \hat{\bar{u}}_{0}\right) \\
-\Gamma \hat{M}\left(\bar{z}_{0}-T \hat{\bar{u}}_{0}\right)
\end{array}\right] .
\end{aligned}
$$

Next, define the limiting cost $J_{\hat{T}, \infty}$ by

$$
J_{\hat{T}, \infty} \triangleq \lim _{k \rightarrow \infty} J\left(\bar{z}_{k}, \hat{\bar{u}}_{k}\right) .
$$

Substituting (5.14) into (3.1) yields

$$
\begin{aligned}
J_{\hat{T}, \infty}=\left(\bar{z}_{0}-T \hat{\bar{u}}_{0}\right)^{\mathrm{T}}\left[Q-Q T \Gamma \hat{M}-(Q T \Gamma \hat{M})^{\mathrm{T}}\right. \\
\left.+\hat{M}^{\mathrm{T}} \Gamma^{\mathrm{T}} D \Gamma \hat{M}\right]\left(\bar{z}_{0}-T \hat{\bar{u}}_{0}\right) .
\end{aligned}
$$

Note that

$$
\left(D^{-1 / 2} T^{\mathrm{T}} Q-D^{1 / 2} \Gamma \hat{M}\right)^{\mathrm{T}}\left(D^{-1 / 2} T^{\mathrm{T}} Q-D^{1 / 2} \Gamma \hat{M}\right) \geqslant 0
$$

and, hence

$$
Q T D^{-1} T^{\mathrm{T}} Q \geqslant Q T \Gamma \hat{M}+(Q T \Gamma \hat{M})^{\mathrm{T}}-\hat{M}^{\mathrm{T}} \Gamma^{\mathrm{T}} D \Gamma \hat{M} .
$$




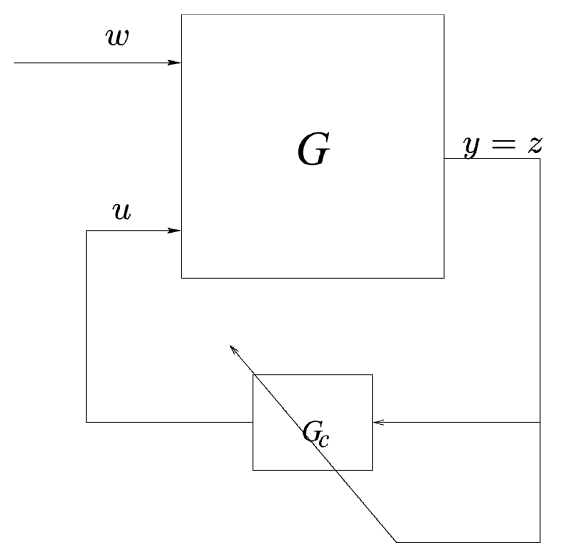

Fig. 2. Adaptive HSS control architecture. The performance variable $z$ is used to determine an estimate $\hat{T}$ of $T$, which is used in the adaptive control law.

Since $z_{0}-T u_{0}=z_{0}-T \hat{\bar{u}}_{0}=W \bar{w}$, it follows from (3.15), (5.16), and (5.18) that

$$
J_{\hat{T}, \infty} \geqslant \mathcal{J}\left(\bar{w}, \bar{u}_{\text {opt }}\right)
$$

which confirms that the performance is not better than the optimal performance obtained when $T$ is exactly known.

\section{ADAPTIVE HSS CONTROL USING RLS}

Here, we discuss online identification of the matrix $T$, which is then used as the basis for an adaptive extension of HSS control (see Fig.2). Define $\Delta \bar{z}_{k} \in \mathbb{R}^{2 p}$ and $\Delta \bar{u}_{k} \in \mathbb{R}^{2 m}$ by

$$
\Delta \bar{z}_{k} \triangleq \bar{z}_{k}-\bar{z}_{k-1}, \quad \Delta \bar{u}_{k} \triangleq \bar{u}_{k}-\bar{u}_{k-1}
$$

and $\Delta Z_{k} \in \mathbb{R}^{2 p \times k}$ and $\Delta U_{k} \in \mathbb{R}^{2 m \times k}$ by

$$
\Delta Z_{k} \triangleq\left[\Delta \bar{z}_{1} \cdots \Delta \bar{z}_{k}\right], \quad \Delta U_{k} \triangleq\left[\Delta \bar{u}_{1} \cdots \Delta \bar{u}_{k}\right] .
$$

The system dynamics (2.16) can be represented by

$$
\Delta \bar{z}_{k}=T \Delta \bar{u}_{k} .
$$

Hence, it follows from (6.1) and (6.3) that

$$
\Delta Z_{k}=T \Delta U_{k} .
$$

Assuming $\Delta U_{k} \Delta U_{k}^{\mathrm{T}}$ is nonsingular, we define

$$
P_{k} \triangleq\left(\Delta U_{k} \Delta U_{k}^{\mathrm{T}}\right)^{-1}
$$

and it follows from (6.4) that the least squares estimate $\hat{T}_{\mathrm{LS}_{k}}$ of $T$ is given by

$$
\hat{T}_{\mathrm{LS}_{k}}=\Delta Z_{k} \Delta U_{k}^{\mathrm{T}} P_{k} .
$$

Hence, substituting (6.4) and (6.4) into (6.5) yields

$$
\hat{T}_{\mathrm{LS}_{k}}=T .
$$

Note that $\Delta Z_{k} \in \mathbb{R}^{2 p \times k}$ and $\Delta U_{k} \in \mathbb{R}^{2 m \times k}$ increase in size as $k$ increases and, thus, the computational burden of evaluating
(6.6) increases as $k$ increases. Hence, we use a recursive procedure that uses the estimate $\hat{T}_{\mathrm{LS}_{k}}$ and the measurements $\Delta \bar{z}_{k}$ and $\Delta \bar{u}_{k}$ to obtain a new estimate $\hat{T}_{\mathrm{LS}_{k+1}}$ of $T$. The recursive least squares method is an efficient procedure for iteratively updating $\hat{T}_{\mathrm{LS}_{k}}$ based on the past and current values of $\Delta \bar{z}_{k}$ and $\Delta \bar{u}_{k}$. From (6.5), it follows that

$$
\begin{aligned}
P_{k+1}^{-1} & =\Delta U_{k+1} \Delta U_{k+1}^{\mathrm{T}}=\sum_{i=1}^{k+1} \Delta \bar{u}_{i} \Delta \bar{u}_{i}^{\mathrm{T}} \\
& =\sum_{i=1}^{k} \Delta \bar{u}_{i} \Delta \bar{u}_{i}^{\mathrm{T}}+\Delta \bar{u}_{k+1} \Delta \bar{u}_{k+1}^{\mathrm{T}} \\
& =P_{k}^{-1}+\Delta \bar{u}_{k+1} \Delta \bar{u}_{k+1}^{\mathrm{T}} .
\end{aligned}
$$

Rewriting (6.6) as

$$
\hat{T}_{\mathrm{LS}_{k}}=\left(\sum_{i=1}^{k} \Delta \bar{z}_{i} \Delta \bar{u}_{i}^{\mathrm{T}}\right) P_{k}
$$

and replacing $k$ by $k+1$ in (6.9) yields

$$
\begin{aligned}
\hat{T}_{\mathrm{LS}_{k+1}} & =\left(\sum_{i=1}^{k+1} \Delta \bar{z}_{i} \Delta \bar{u}_{i}^{\mathrm{T}}\right) P_{k+1} \\
& =\left(\sum_{i=1}^{k} \Delta \bar{z}_{i} \Delta \bar{u}_{i}^{\mathrm{T}}+\Delta \bar{z}_{k+1} \Delta \bar{u}_{k+1}^{\mathrm{T}}\right) P_{k+1} .
\end{aligned}
$$

Hence, substituting $P_{k}^{-1}$ from (6.8) into (6.9) yields

$$
\sum_{i=1}^{k} \Delta \bar{z}_{i} \Delta \bar{u}_{i}^{\mathrm{T}}=\hat{T}_{\mathrm{LS}_{k}}\left(P_{k+1}^{-1}-\Delta \bar{u}_{k+1} \Delta \bar{u}_{k+1}^{\mathrm{T}}\right) .
$$

Substituting (6.11) into (6.10) yields

$$
\begin{aligned}
\hat{T}_{\mathrm{LS}_{k+1}}= & {\left[\hat{T}_{\mathrm{LS}_{k}}\left(P_{k+1}^{-1}-\Delta \bar{u}_{k+1} \Delta \bar{u}_{k+1}^{\mathrm{T}}\right)+\Delta \bar{z}_{k+1} \Delta \bar{u}_{k+1}^{\mathrm{T}}\right] } \\
& \times P_{k+1} \\
= & \hat{T}_{\mathrm{LS}_{k}}+\left[\Delta \bar{z}_{k+1}-\hat{T}_{\mathrm{LS}_{k}} \Delta \bar{u}_{k+1}\right] \Delta \bar{u}_{k+1}^{\mathrm{T}} P_{k+1} \\
= & \hat{T}_{\mathrm{LS}_{k}}+\varepsilon_{k+1} K_{k+1}
\end{aligned}
$$

where

$$
\varepsilon_{k+1} \triangleq \Delta \bar{z}_{k+1}-\hat{T}_{\mathrm{LS}_{k}} \Delta \bar{u}_{k+1}
$$

and

$$
K_{k+1} \triangleq \Delta \bar{u}_{k+1}^{\mathrm{T}} P_{k+1} .
$$

Using the matrix inversion lemma [22] in (6.8), $P_{k+1}$ can be expressed recursively as

$$
\begin{aligned}
P_{k+1}=P_{k}- & P_{k} \Delta \bar{u}_{k+1} \\
& \times\left(1+\Delta \bar{u}_{k+1}^{\mathrm{T}} P_{k} \Delta \bar{u}_{k+1}\right)^{-1} \Delta \bar{u}_{k+1}^{\mathrm{T}} P_{k} .
\end{aligned}
$$

Combining (6.14) and (6.15) yields

$$
K_{k+1}=\left(1+\Delta \bar{u}_{k+1}^{\mathrm{T}} P_{k} \Delta \bar{u}_{k+1}\right)^{-1} \Delta \bar{u}_{k+1}^{\mathrm{T}} P_{k} .
$$


Since $\Delta \bar{u}_{i} \Delta \bar{u}_{i}^{\mathrm{T}}$ is positive semidefinite for all $i=1,2, \ldots$, (6.8) implies that if $\Delta U_{k_{0}} \Delta U_{k_{0}}^{\mathrm{T}}$ is nonsingular, then $\Delta U_{k} \Delta U_{k}^{\mathrm{T}}$ is nonsingular for all $k>k_{0}$. Hence, the recursive procedure for determining $T_{\mathrm{LS}_{k}}$ for all $k>k_{0}$ is given by

$$
\begin{aligned}
K_{k+1} & =\left(1+\Delta \bar{u}_{k+1}^{\mathrm{T}} P_{k} \Delta \bar{u}_{k+1}\right)^{-1} \Delta \bar{u}_{k+1}^{\mathrm{T}} P_{k} \\
\hat{T}_{\mathrm{LS}} & =\hat{T}_{\mathrm{LS}_{k}}+\varepsilon_{k+1} K_{k+1} \\
P_{k+1} & =P_{k}\left(I-\Delta \bar{u}_{k+1} K_{k+1}\right)
\end{aligned}
$$

Note that

$$
\operatorname{rank}\left(\Delta U_{k} \Delta U_{k}^{\mathrm{T}}\right)=\operatorname{rank}\left(\Delta U_{k}\right) \leqslant \min (k, 2 m) .
$$

Since $\Delta U_{k} \Delta U_{k}^{\mathrm{T}}$ is $2 \mathrm{~m} \times 2 \mathrm{~m}$, it follows from (6.20) that $\Delta U_{k} \Delta U_{k}^{\mathrm{T}}$ is singular for all $k<2 m$. Hence, the recursive procedure (6.17)-(6.19) cannot be used for $k<2 m$.

\section{AdAPTIVE HSS CONTROL Using PSEUdo-RLS}

A suboptimal estimate $\hat{T}_{k}$ of $T$ can be obtained by replacing $P_{k}$ in (6.17)-(6.19) by $\hat{P}_{k}$, that is

$$
\begin{aligned}
K_{k+1} & =\left(1+\Delta \bar{u}_{k+1}^{\mathrm{T}} \hat{P}_{k} \Delta \bar{u}_{k+1}\right)^{-1} \Delta \bar{u}_{k+1}^{\mathrm{T}} \hat{P}_{k} \\
\hat{T}_{k+1} & =\hat{T}_{k}+\hat{\varepsilon}_{k+1} K_{k+1} \\
\hat{P}_{k+1} & =\hat{P}_{k}\left(I-\Delta \bar{u}_{k+1} K_{k+1}\right)
\end{aligned}
$$

where $\hat{P}_{0}$ is positive definite but otherwise arbitrary, and $\hat{\varepsilon}_{k+1}$ is defined by

$$
\hat{\varepsilon}_{k+1} \triangleq \Delta \bar{z}_{k+1}-\hat{T}_{k} \Delta \bar{u}_{k+1} .
$$

Note that (7.1)-(7.3) can be started with an arbitrary initial estimate $\hat{T}_{0}$ of $T$. It follows from (7.1)-(7.3) that $\hat{P}_{k}$ is positive definite for all $k \geqslant 0$ and is given by

$$
\hat{P}_{k}=\left(\hat{P}_{0}^{-1}+\Delta U_{k} \Delta U_{k}^{\mathrm{T}}\right)^{-1} .
$$

Note that substituting (6.13), (7.1), and (7.3) into (7.2) yields

$$
\hat{T}_{k+1}=\hat{T}_{k}+\hat{\varepsilon}_{k} \Delta \bar{u}_{k+1}^{\mathrm{T}} \hat{P}_{k+1} .
$$

Multiplying (7.6) by $\hat{P}_{k}^{-1}$ yields

$$
\hat{T}_{k+1} \hat{P}_{k+1}^{-1}=\hat{T}_{k}\left(\hat{P}_{k+1}^{-1}-\Delta \bar{u}_{k+1} \Delta \bar{u}_{k+1}^{\mathrm{T}}\right)+\Delta \bar{z}_{k+1} \Delta \bar{u}_{k+1}^{\mathrm{T}} .
$$

Hence, it follows from (7.5) and (7.7) that, for all $k>0, \hat{T}_{k}$ is given by

$$
\hat{T}_{k}=\left(\hat{T}_{0} \hat{P}_{0}^{-1}+\Delta Z_{k} \Delta U_{k}^{\mathrm{T}}\right)\left(\hat{P}_{0}^{-1}+\Delta U_{k} \Delta U_{k}^{\mathrm{T}}\right)^{-1} .
$$

Since $\hat{P}_{0}$ is positive definite, the inverse in (7.8) always exists, and hence, the recursive procedure can be used for all $k \geqslant 0$. The updated estimate $\hat{T}_{k}$ is used at each control update step to calculate the control law $\bar{u}_{k+1}$, which is given by

$$
\bar{u}_{k+1}=-\hat{M}_{k}\left(\bar{z}_{k}-\hat{T}_{k} \bar{u}_{k}\right)
$$

where $\hat{M}_{k}$ is defined by

$$
\hat{M}_{k} \triangleq\left(\hat{T}_{k}^{\mathrm{T}} Q \hat{T}_{k}+S^{\mathrm{T}} \hat{T}_{k}+\hat{T}_{k}^{\mathrm{T}} S+R\right)^{-1}\left(\hat{T}_{k}^{\mathrm{T}} Q+S^{\mathrm{T}}\right) .
$$

Let $k$ be such that $\Delta U_{k} \Delta U_{k}^{\mathrm{T}}$ is nonsingular. Then it follows from (7.5) that $\hat{P}_{k}-\left(\Delta U_{k} \Delta U_{k}^{\mathrm{T}}\right)^{-1} \rightarrow 0$ as $\hat{P}_{0} \rightarrow \infty$, and hence, (6.4) and (7.8) imply that $\hat{T}_{k} \rightarrow T$ as $\hat{P}_{0} \rightarrow \infty$.

Next, we show that, for all positive-definite $\hat{P}_{0}, \hat{T}_{k}$ converges as $k \rightarrow \infty$. Define $\Delta T_{k} \in \mathbb{R}^{2 p \times 2 m}$ by

$$
\Delta T_{k} \triangleq \hat{T}_{k}-T
$$

where $\hat{T}_{k}$ is updated using (7.1)-(7.3). Substituting (6.4) into (7.8) yields

$$
\hat{T}_{k}=\left(\hat{T}_{0} \hat{P}_{0}^{-1}+T \Delta U_{k} \Delta U_{k}^{\mathrm{T}}\right)\left(\hat{P}_{0}^{-1}+\Delta U_{k} \Delta U_{k}^{\mathrm{T}}\right)^{-1} .
$$

Post-multiplying (7.12) by $\hat{P}_{0}^{-1}+\Delta U_{k} \Delta U_{k}^{\mathrm{T}}$ yields

$$
\hat{T}_{k}\left(\hat{P}_{0}^{-1}+\Delta U_{k} \Delta U_{k}^{\mathrm{T}}\right)=\hat{T}_{0} \hat{P}_{0}^{-1}+T \Delta U_{k} \Delta U_{k}^{\mathrm{T}} .
$$

Subtracting $T\left(\hat{P}_{0}^{-1}+\Delta U_{k} \Delta U_{k}^{\mathrm{T}}\right)$ from both sides of (7.13) and then substituting (7.5) into the resulting expression yields

$$
\Delta T_{k}=\Delta T_{0} \hat{P}_{0}^{-1} \hat{P}_{k} .
$$

For all $k$

$$
\hat{P}_{k+1}^{-1}=\hat{P}_{k}^{-1}+\Delta \bar{u}_{k+1} \Delta \bar{u}_{k+1}
$$

which implies that

$$
\hat{P}_{k+1}^{-1} \geqslant \hat{P}_{k}^{-1}
$$

and, hence

$$
\hat{P}_{k+1} \leqslant \hat{P}_{k} \text {. }
$$

Furthermore, since $\hat{P}_{0}$ is positive definite, it follows from (7.17) that, for all $k \geqslant 0$

$$
\hat{P}_{k}>0 \text {. }
$$

It follows from (7.17) and (7.18) that $\hat{P}_{k}$ is a nonincreasing sequence that is bounded from below. Hence, $\lim _{k \rightarrow \infty} \hat{P}_{k}$ exists. Next, define $\hat{P}_{\infty}$ by

$$
\hat{P}_{\infty} \triangleq \lim _{k \rightarrow \infty} \hat{P}_{k}
$$

Taking the limit as $k \rightarrow \infty$ of (7.14) yields

$$
\lim _{k \rightarrow \infty} \Delta T_{k}=\Delta T_{0} \hat{P}_{0}^{-1}, \lim _{k \rightarrow \infty} \hat{P}_{k}=\Delta T_{0} \hat{P}_{0}^{-1} \hat{P}_{\infty} .
$$

Hence, it follows from (7.11) that $\lim _{k \rightarrow \infty} \hat{T}_{k}$ exists, that is, $\hat{T}_{k}$ converges. However, there is no guarantee that $\hat{T}_{k}$ converges to $T$. For example, if $\bar{u}_{0}=0, S=0$, and $\hat{T}_{0}=0$, then (7.9) implies that $\bar{u}_{1}=0$. Hence, it follows from (7.1)-(7.3), that $\hat{T}_{k}=0$ and $\hat{P}_{k}=\hat{P}_{0}$ for all $k \geqslant 0$. In the next section, we 
introduce a persistent excitation condition that guarantees convergence of $\hat{T}_{k}$ to $T$.

Note that substituting (2.13) into (7.9) yields

$$
\Delta \bar{u}_{k+1}=\left(\hat{M}_{k} \Delta T_{k}-I\right) \bar{u}_{k}-\hat{M}_{k} W \bar{w} .
$$

Without any loss of generality, let $u_{0}=0$, so that for all $k \geqslant 1$

$$
\bar{u}_{k}=\sum_{i=1}^{k} \Delta \bar{u}_{i}
$$

Substituting (7.14) and (7.22) into (7.21) yields

$$
\begin{aligned}
\Delta \bar{u}_{k+1}= & {\left[\Phi_{k}\left(\hat{P}_{0}^{-1}+\sum_{i=1}^{k} \Delta \bar{u}_{i} \Delta \bar{u}_{i}^{\mathrm{T}}\right)^{-1}-I\right] } \\
& \times\left(\sum_{i=1}^{k} \Delta \bar{u}_{i}\right)+b_{k}
\end{aligned}
$$

where $\Phi_{k}$ and $b_{k}$ are defined by

$$
\Phi_{k} \triangleq \hat{M}_{k} \Delta T_{0} \hat{P}_{0}^{-1}, b_{k} \triangleq-\hat{M}_{k} W \bar{w} .
$$

Note that (7.21) and (7.22) imply that

$$
\begin{aligned}
\bar{u}_{k+1}= & \Phi_{k}\left(\hat{P}_{0}^{-1}+\sum_{i=1}^{k}\left(\bar{u}_{i}-\bar{u}_{i-1}\right)\left(\bar{u}_{i}-\bar{u}_{i-1}\right)^{\mathrm{T}}\right)^{-1} \\
& \times \bar{u}_{k}+b_{k} .
\end{aligned}
$$

Since $\lim _{k \rightarrow \infty} \hat{T}_{k}$ exists, $\lim _{k \rightarrow \infty} \Phi_{k}$ and $\lim _{k \rightarrow \infty} b_{k}$ exist. Numerical simulations of (7.23) and (7.25) suggest that, for all positive-definite $\hat{P}_{0}$

$$
\lim _{k \rightarrow \infty} \Delta \bar{u}_{k}=0
$$

and $\lim _{k \rightarrow \infty} \bar{u}_{k}$ exists, which implies that $\bar{u}_{k}$ is bounded. Hence, (2.13) implies that $z_{k}$ is also bounded.

\section{AdAPTIVE HSS CONTROL With Persistent Excitation}

The convergence of $\hat{T}_{k}$ is guaranteed by the adaptive HSS algorithm, and numerical simulations suggest that $\bar{u}_{k}$ and $\bar{z}_{k}$ are bounded. However, the steady-state performance of the adaptive HSS control depends on the steady-state value of the estimate $\hat{T}_{k}$. It follows from (7.11) and (7.14) that

$$
\hat{T}_{k}=T+\Delta T_{0} \hat{P}_{0}^{-1} \hat{P}_{k} .
$$

Consequently, if $\hat{P}_{k} \rightarrow 0$ as $k \rightarrow \infty$, then $\hat{T}_{k} \rightarrow T$ as $k \rightarrow \infty$. It follows from (6.2) and (7.5) that

$$
\hat{P}_{k}=\left(\hat{P}_{0}^{-1}+\sum_{i=1}^{k} \Delta \bar{u}_{i} \Delta \bar{u}_{i}^{\mathrm{T}}\right)^{-1} .
$$

Hence, if

$$
\lim _{k \rightarrow \infty} \sigma_{\min }\left(\sum_{i=1}^{k} \Delta \bar{u}_{i} \Delta \bar{u}_{i}^{\mathrm{T}}\right)=\infty
$$

then (8.2) implies that $\hat{P}_{k} \rightarrow 0$ as $k \rightarrow \infty$. A sufficient condition for (8.3) is that $\Delta \bar{u}_{k}$ be persistently exciting [22], that is, there

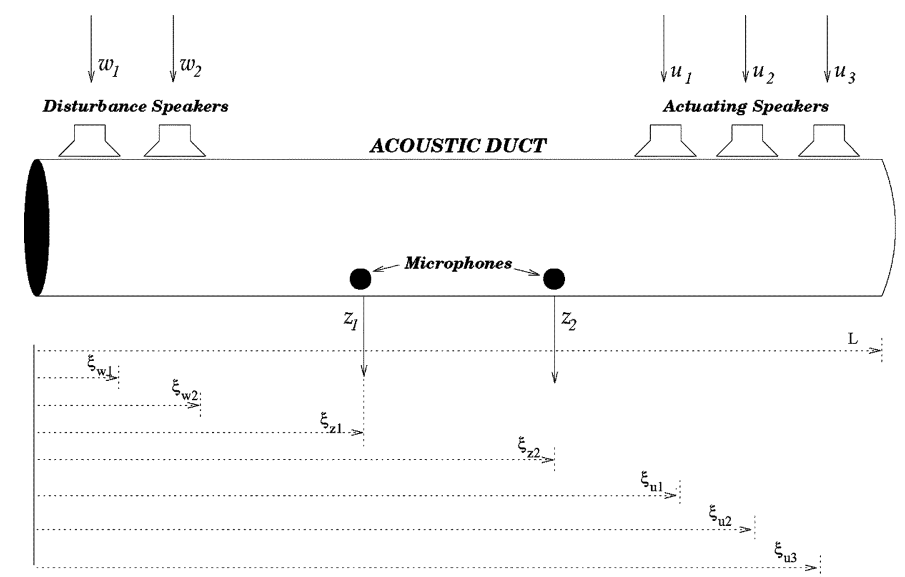

Fig. 3. Acoustic duct.

exists $\rho>0$ such that, for all $n \geqslant 2 m$

$$
\frac{1}{2 m} \sum_{i=n}^{n+2 m-1} \Delta \bar{u}_{i} \Delta \bar{u}_{i}^{\mathrm{T}}>\rho I .
$$

However, since $\bar{u}_{k}$ is given by the adaptive control law (7.9), $\Delta \bar{u}_{k}$ may not be persistently exciting. Hence, a modified control law is needed to ensure that (8.3) holds.

Note that the adaptive control law in (7.9) can be expressed as $\bar{u}_{k+1}=\bar{u}_{k}+\Delta \bar{u}_{k}$, where

$$
\Delta \bar{u}_{k}=-\hat{M}_{k} \bar{z}_{k}+\left(\hat{M}_{k} \hat{T}_{k}-I\right) \bar{u}_{k} .
$$

Next, for $n=0,1, \ldots$, and for all $k=n(2 m)+k_{0}+1, \ldots,(n+$ 1) $(2 m)+k_{0}$, define $\tilde{\delta}_{k} \in \mathbb{R}^{2 m}$ by

$$
\tilde{\delta}_{k} \triangleq\left[\begin{array}{lll}
0_{1 \times i-1} & \delta_{n} & 0_{1 \times 2 m-i}
\end{array}\right]^{\mathrm{T}}
$$

where $i=k-n(2 m)+k_{0}$. Next, define $\Delta \tilde{u}_{k}$ by

$$
\Delta \tilde{u}_{k} \triangleq \Delta \bar{u}_{k}+\tilde{\delta}_{k} .
$$

Note that

$$
\sum_{i=2 m n+k_{0}+1}^{2 m n+k_{0}+2 m} \Delta \tilde{u}_{i} \Delta \tilde{u}_{i}^{\mathrm{T}}=\Delta \tilde{U}\left(k_{0}, n\right) \Delta \tilde{U}^{\mathrm{T}}\left(k_{0}, n\right)
$$

where $\Delta \tilde{U}\left(k_{0}, n\right) \in \mathbb{R}^{2 m \times 2 m}$ is defined by

$$
\Delta \tilde{U}\left(k_{0}, n\right) \triangleq\left[\Delta \tilde{u}_{2 m n+k_{0}+1} \cdots \Delta \tilde{u}_{2 m n+k_{0}+2 m}\right] .
$$

It follows from (8.6), (8.7), and (8.9) that

$$
\Delta \tilde{U}\left(k_{0}, n\right)=\bar{U}\left(k_{0}, n\right)+\delta_{n} I_{2 m}
$$

where $\bar{U}\left(k_{0}, n\right) \in \mathbb{R}^{2 m \times 2 m}$ is defined by (8.9) with $\Delta \tilde{u}_{k}$ replaced by $\Delta \bar{u}_{k}$. Hence, if the sequence of $\delta_{i}$ 's is chosen so that

$$
\lim _{n \rightarrow \infty} \sigma_{l}^{2} \Delta \tilde{U}\left(k_{0}, n\right)=\infty
$$

then (8.8) implies that $\Delta \tilde{u}_{k}$ is persistently exciting. The modified control law is given by

$$
\bar{u}_{k+1}=\bar{u}_{k}+\Delta \tilde{u}_{k}
$$


TABLE I

NOtATION FOR SimUlated NoISE CANCELLATION IN AN ACOUSTIC DUCT

\begin{tabular}{|c|c|}
\hline$c$ & phase speed of acoustic wave $\left(343 \mathrm{~m} / \mathrm{s}\right.$ in air $\left.{ }^{a}\right)$ \\
\hline$v_{w}(t)$ & speaker cone velocity of disturbance speaker \\
\hline$v_{u}(t)$ & speaker cone velocity of actuating speaker \\
\hline$\rho_{0}$ & equilibrium density of air $\left(1.21 \mathrm{~kg} / \mathrm{m}^{3}{ }^{a}\right)$ \\
\hline$\zeta_{i}$ & damping ratio of $i$ th acoustic mode ( 0.01 in this example) \\
\hline$r$ & number of modal frequencies retained \\
\hline$d$ & number of disturbance speakers \\
\hline$m$ & number of actuating speakers \\
\hline$p$ & number of output microphones \\
\hline$A_{\mathrm{s}}$ & cross sectional area of speaker \\
\hline$L$ & length of the acoustic duct \\
\hline$\xi_{w i}$ & location of $i$ th disturbance speaker \\
\hline$\xi_{u i}$ & location of $i$ th actuating speaker \\
\hline$\xi_{z i}$ & location of $i$ th microphone \\
\hline
\end{tabular}

$a$ at room conditions.

where $\Delta \tilde{u}_{k}$ is given by (8.7). If the performance is satisfactory for some $k=k_{\mathrm{s}}$, then $\tilde{\delta}_{k}$ in (8.7) is set to zero, that is, $\tilde{\delta}_{k}=0$ for all $k \geqslant k_{\mathrm{s}}$ and, thus, $\Delta \tilde{u}_{k}=\Delta \bar{u}_{k}$ for all $k \geqslant k_{\mathrm{s}}$.

\section{Simulation Example: Noise CanCellation IN AN ACOUSTIC DUCT}

The equation of motion for the acoustic duct shown in Fig. 3, is given by

$$
\begin{aligned}
\frac{1}{c^{2}} p_{t t}(\xi, t)= & p_{\xi \xi}(\xi, t)+\rho_{0} \dot{v}_{u}(t) \delta\left(\xi-\xi_{u}\right) \\
& +\rho_{0} \dot{v}_{w}(t) \delta\left(\xi-\xi_{w}\right) \\
z_{x}(t)= & p\left(\xi_{z}, t\right)
\end{aligned}
$$

where the notation is defined in Table I (see [1]).

By separation of variables with $r$ modes, $p(\xi, t)$ is given by

$$
p(\xi, t)=\sum_{k=0}^{r} q_{k}(t) V_{k}(\xi)
$$

where $q_{k}(t)$ are modal coordinates. The state space realization of (9.1) of order $2 r$ is given by

$$
\begin{aligned}
\dot{x}(t) & =A x(t)+B u(t)+D_{1} w(t) \\
z(t) & =C x(t)
\end{aligned}
$$

where

$$
\begin{aligned}
& x(t) \triangleq\left[\begin{array}{lllll}
\int_{0}^{t} q_{1}(\sigma) d \sigma & q_{1}(t) & \cdots & \int_{0}^{t} q_{r}(\sigma) d \sigma & q_{r}(t)
\end{array}\right]^{\mathrm{T}} \\
& u(t) \triangleq\left[\begin{array}{lll}
A_{\mathrm{s}} v_{u 1}(t) & \cdots & A_{\mathrm{s}} v_{u m}(t)
\end{array}\right]^{\mathrm{T}} \\
& w(t) \triangleq\left[\begin{array}{lll}
A_{\mathrm{s}} v_{w 1}(t) & \cdots & A_{\mathrm{s}} v_{w d}(t)
\end{array}\right]^{\mathrm{T}}
\end{aligned}
$$

and

$$
\begin{aligned}
& A \triangleq\left[\begin{array}{ccccc}
0 & 1 & & & \\
-\omega_{n 1}^{2} & -2 \zeta_{1} \omega_{n 1} & & & \\
& & \ddots & & \\
& & & 0 & 1 \\
& & & -\omega_{n r}^{2} & -2 \zeta_{r} \omega_{n r}
\end{array}\right] \\
& \begin{array}{r}
B \triangleq\left[\begin{array}{ccc}
0 & \cdots & 0 \\
\frac{\rho_{0}}{A_{\mathrm{s}}} V_{1}\left(\xi_{u 1}\right) & \cdots & \frac{\rho_{0}}{A_{\mathrm{s}}} V_{1}\left(\xi_{u m}\right) \\
\vdots & \vdots & \vdots \\
0 & \cdots & 0 \\
\frac{\rho_{0}}{A_{\mathrm{s}}} V_{r}\left(\xi_{u 1}\right) & \cdots & \frac{\rho_{0}}{A_{\mathrm{s}}} V_{r}\left(\xi_{u m}\right)
\end{array}\right] \\
D_{1} \triangleq\left[\begin{array}{ccc}
\frac{\rho_{0}}{A_{\mathrm{s}}} V_{1}\left(\xi_{w 1}\right) & \cdots & \frac{\rho_{0}}{A_{\mathrm{s}}} V_{1}\left(\xi_{w d}\right) \\
\vdots & \vdots & \vdots \\
0 & \cdots & 0 \\
\frac{\rho_{0}}{A_{\mathrm{s}}} V_{r}\left(\xi_{w 1}\right) & \cdots & \frac{\rho_{0}}{A_{\mathrm{s}}} V_{r}\left(\xi_{w d}\right)
\end{array}\right]
\end{array} \\
& C \triangleq\left[\begin{array}{ccccc}
0 & V_{1}\left(\xi_{z 1}\right) & \cdots & 0 & V_{r}\left(\xi_{z 1}\right) \\
\vdots & \vdots & \vdots & \vdots & \vdots \\
0 & V_{1}\left(\xi_{z p}\right) & \cdots & 0 & V_{r}\left(\xi_{z p}\right)
\end{array}\right]
\end{aligned}
$$

where for all $i=1, \ldots, r$

$$
\omega_{n i} \triangleq \frac{i \pi}{L} c, \quad V_{i}(\xi) \triangleq c \sqrt{\frac{2}{L}} \sin \left(\frac{i \pi}{L} \xi\right) .
$$

The acoustic duct is assumed to have two disturbance speakers and three actuation speakers, which are noncolocated. The output is measured using two microphones placed at arbitrary locations. The disturbance signals consist of harmonics at 100 and $150 \mathrm{~Hz}$. Six modal frequencies are retained, and hence, the plant is 12 th order.

HSS control is simulated for the duct. Fig. 4 shows the output from microphone 1 with HSS control started at $t=1 \mathrm{~s}$ with knowledge of $T$. We update $\bar{u}_{k} \in \mathbb{R}^{12}$ every $1 \mathrm{~s}$ using (4.3) and, hence, for all $k<t \leqslant k+1, u(t)$ is given by

$$
\begin{aligned}
u(t)= & \sum_{i=1}^{m}\left[\bar{u}_{2(i-1)+1, k} \sin \left(\omega_{1} t\right)+\bar{u}_{2 i, k} \cos \left(\omega_{1} t\right)\right] \\
& +\sum_{i=1}^{m}\left[\bar{u}_{2 m+2(i-1)+1, k} \sin \left(\omega_{2} t\right)+\bar{u}_{2 m+2 i, k} \cos \left(\omega_{2} t\right)\right]
\end{aligned}
$$

where $\omega_{1}=200 \pi \mathrm{rad} / \mathrm{s}$ and $\omega_{2}=300 \pi \mathrm{rad} / \mathrm{s}$, and, for $i=$ $1, \ldots, 12, \bar{u}_{i, k}$ is the $i$ th entry of $\bar{u}_{k}$.

Although the steady-state performance is satisfactory, the sudden change in the amplitudes of the sinusoids $\bar{u}_{k}$ at the switching instants causes large transients. To reduce the transients, we introduce the interpolating control law

$$
\hat{u}_{k}= \begin{cases}\left(\bar{u}_{k}-\bar{u}_{k-1}\right)\left(\frac{1-e^{\alpha(t-k)}}{1-e^{\alpha t} \delta}\right)+\bar{u}_{k}, & \text { if } k<t \leqslant k+t_{\delta} \\ \bar{u}_{k}, & \text { if } k+t_{\delta}<t<k+1\end{cases}
$$

where $\alpha>0,0<t_{\delta}<1$, and $\bar{u}_{k}$ is updated by using (4.3). The interpolated control input $u(t)$ is given by $(9.5)$ with $\bar{u}_{k}$ replaced by $\hat{u}_{k}$. Since the change in the amplitude of $u(t)$ from $t=k$ to 


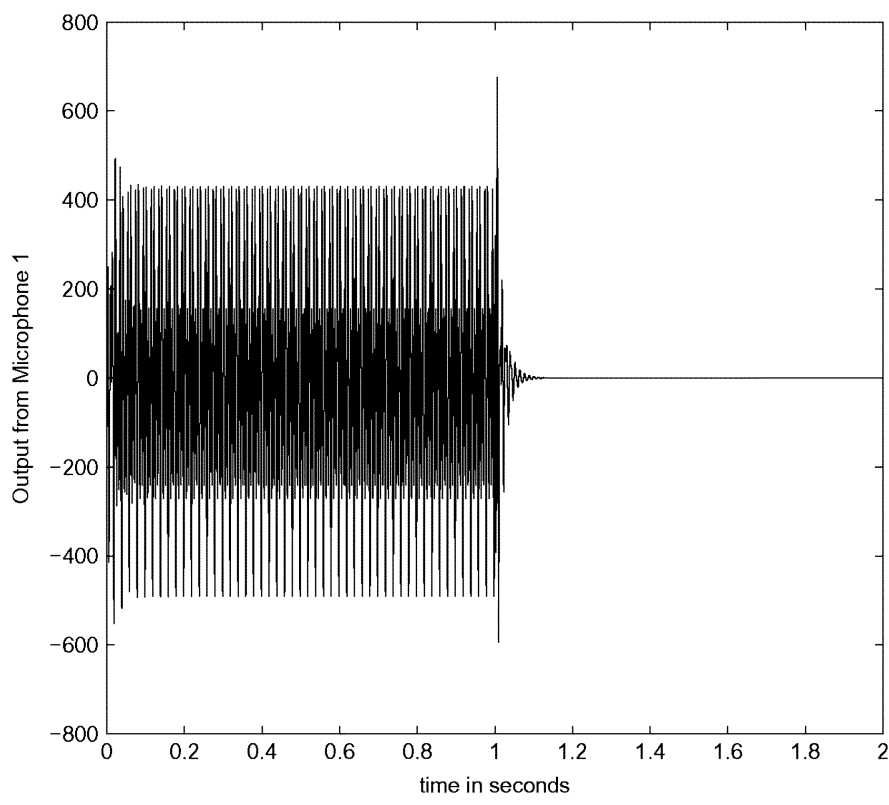

Fig. 4. Simulated output of microphone 1 using HSS control for active noise cancellation. HSS control begins at $t=1 \mathrm{~s}$, resulting in one-step reduction in the output amplitude. The large transient is due to the sudden change in the amplitude of the control input.

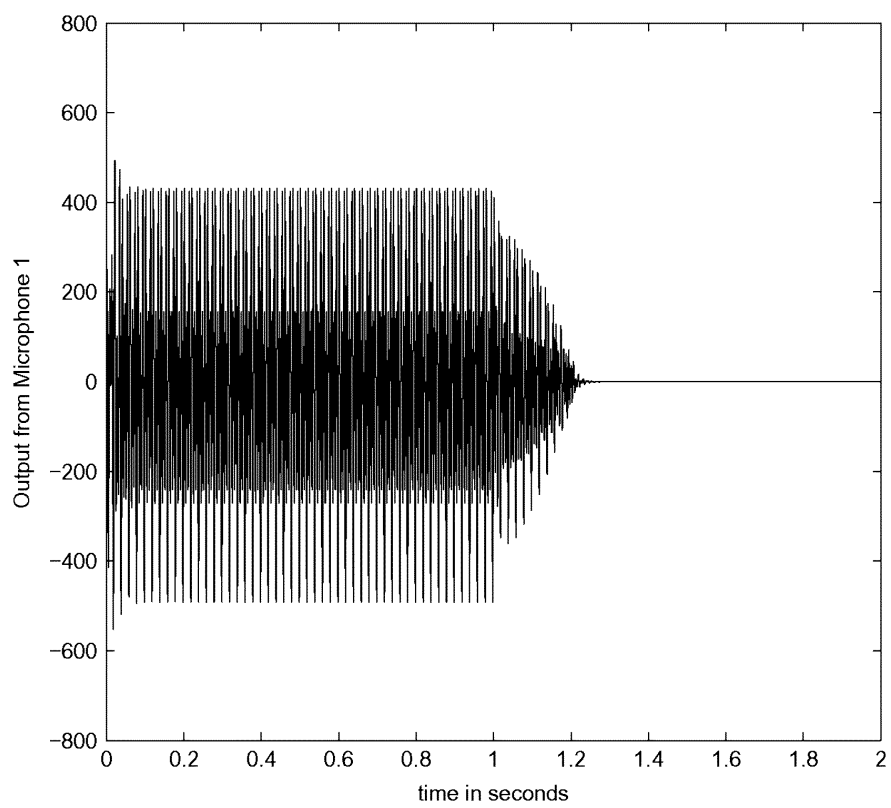

Fig. 5. Simulated output of microphone 1 using HSS control with the interpolating control (9.6). The interpolating control improves the transient performance by interpolating the amplitude of the control input between switching instants.

$t=k+1$ is gradual, the transient performance improves. The output of microphone 1 with the interpolating control is shown in Fig. 5.

Next, at $t=2 \mathrm{~s}$ (see Fig. 6), the microphones are moved to different locations, resulting in a sudden change in the system dynamics as given by the matrix $T$. Since the HSS control algorithm has no knowledge of the change in $T$, there is an increase in the output level. Alternatively, at $t=7 \mathrm{~s}$ (indicated by the vertical dash-dot line), the adaptive HSS control algorithm is used, and the disturbance level is reduced, although not

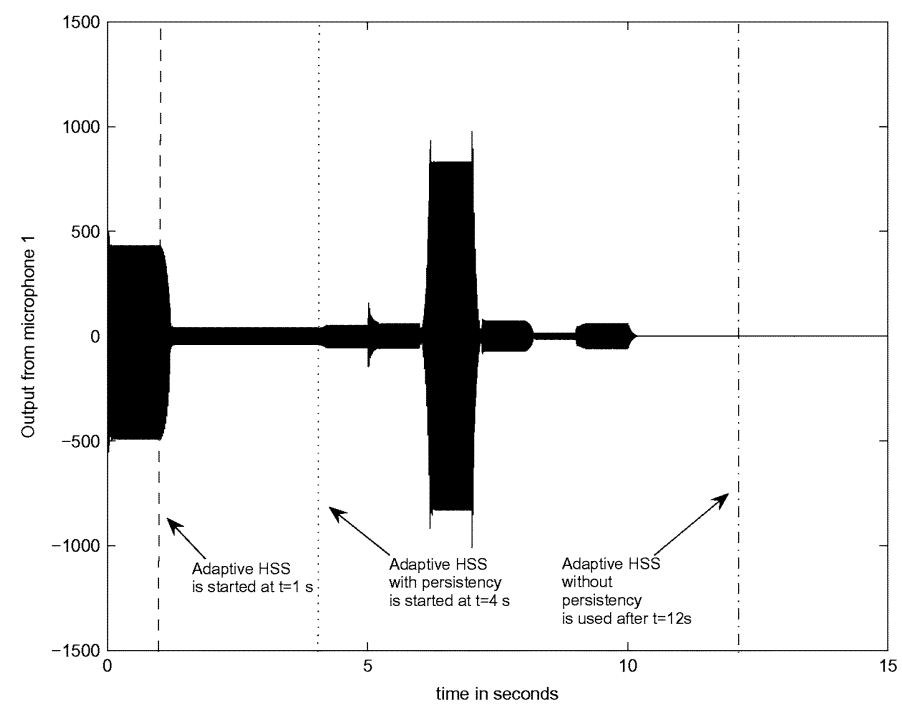

Fig. 6. Simulated disturbance rejection using adaptive HHS control with persistent excitation. Adaptive HSS control without persistent excitation is started at $t=1 \mathrm{~s}$ with initial estimate $\hat{T}_{0}$ of $\mathrm{T}$. At $t=4 \mathrm{~s}$, adaptive HSS control with persistent excitation is used, and at $t=12 \mathrm{~s}$, the performance is satisfactory and, hence, adaptive HSS without persistent excitation is used after $12 \mathrm{~s}$.

in a single control update step. The dashed lines show the diverging envelope of the nonadaptive HSS response. During the time interval within which adaptive HSS is used, we use the interpolating control (9.6), where $\bar{u}_{k}$ is updated using (7.9).

To illustrate the performance of the persistently exciting control law (8.12), the adaptive HSS algorithm without persistent excitation is started at $t=1 \mathrm{~s}$ with an initial estimate $\hat{T}_{0}$ of $T$, such that

$$
\hat{T}_{0}=\left[\begin{array}{llllllllllll}
T_{1} & T_{2} & 0 & T_{4} & T_{5} & T_{6} & 0 & 0 & T_{9} & T_{10} & 0 & T_{12}
\end{array}\right]
$$

where $T_{i}$ is the $i$ th column of $T$. Since $\Delta \bar{u}_{k}$ is not persistently exciting, the estimate $\hat{T}_{k}$ does not converge to $T$ and, hence, the performance is poor (see Fig. 7). At $t=4 \mathrm{~s}$, adaptive HSS with persistent excitation is used with $\delta_{n}=0.01$ for all $n$. The numerical results indicate that $\hat{T}_{k}$ approaches $T$, which suggests that $\Delta \tilde{u}_{k}$ is persistently exciting. Since the performance is satisfactory at $t=12 \mathrm{~s}, \delta_{k}$ is set to 0 and adaptive HSS without persistent excitation is used for all $t \geqslant 12 \mathrm{~s}$. Since adaptive HSS with persistent excitation is used instead of the optimal control input between $t=4 \mathrm{~s}$ and $t=12 \mathrm{~s}$, the microphone output level increases.

\section{EXPERIMENTAl EXAMPLE: Noise CANCELlation IN AN ACOUSTIC DRUM}

Although the equations of motion for the acoustic drum are different from those of the acoustic duct, the problem of disturbance rejection is identical from the point of view of HSS control. In the previous case, sound waves propagate along the length of the duct, and hence, the duct is essentially a spatially one-dimensional (1-D) system (see [1]). This assumption is not valid for an acoustic drum which has longitudinal, transverse, and circumferential modes. As with the duct, however, the input-output response is linear and, hence, HSS control can be used to reject a disturbance with a known harmonic spectrum. 


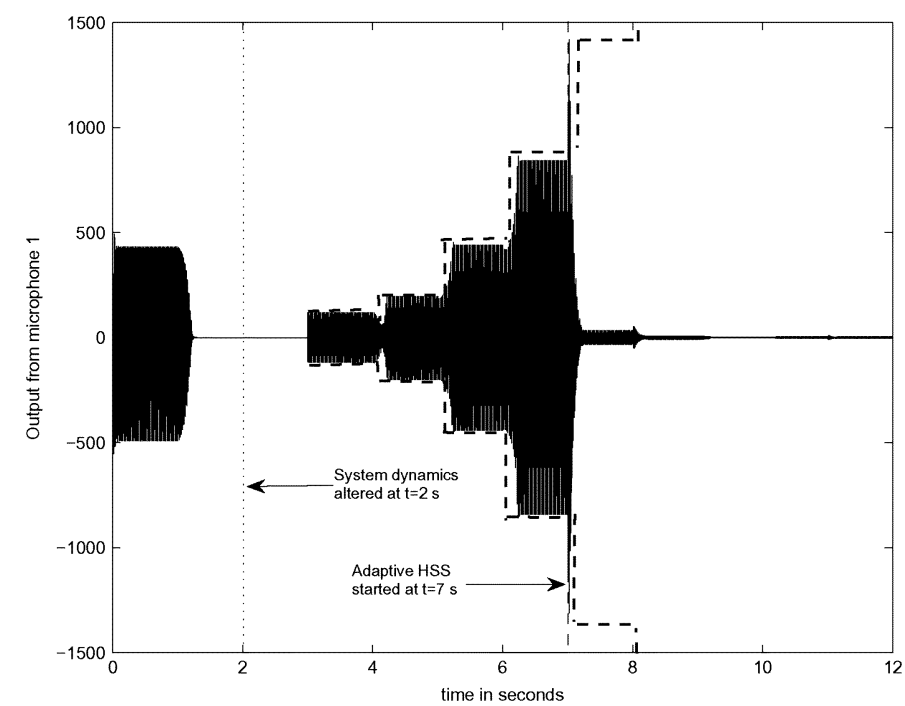

Fig. 7. Simulated disturbance rejection using adaptive HHS control. Microphone 1 is moved to a new location at $t=2 \mathrm{~s}$ and the HSS response is unbounded (shown by the dashed envelope). Alternatively, adaptive HSS control is applied at $t=7 \mathrm{~s}$, and disturbance rejection is achieved. The interpolating control (9.6) is used for improved transient performance.

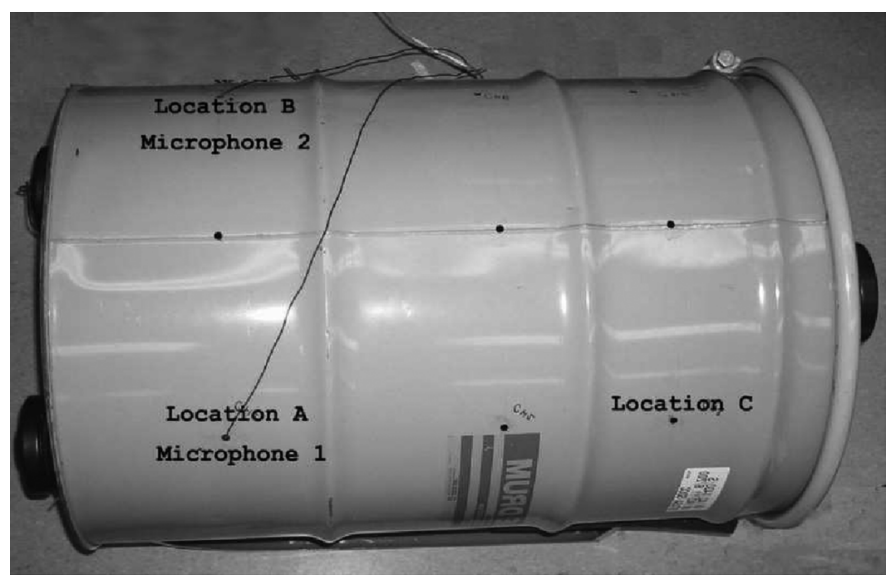

Fig. 8. Top view of the acoustic drum with two end-mounted actuating speakers, one disturbance speaker, and two internal microphones. Microphone 1 is initially at location A and is moved to location C, while the HSS control algorithm is operating. Microphone 2 is placed at location B throughout the experiment.

The acoustic drum (see Fig. 8) has two end-mounted speakers on both sides, while microphones can be suspended inside the drum through holes drilled along the top. The dimensions of the acoustic drum are given in Table II.

A constant-amplitude, single-tone disturbance signal $w(t) \in$ $\mathbb{R}$ with frequency $50 \mathrm{~Hz}(\omega=100 \pi \mathrm{rad} / \mathrm{s})$ is produced by one disturbance speaker. The two actuation speakers on the other end of the drum produce the control $u(t) \in \mathbb{R}^{2}$ for cancelling the disturbance. The control objective is to reduce the output $z(t) \in \mathbb{R}^{2}$ measured by microphones 1 and 2 at locations $A$ and $\mathrm{B}$, respectively. We use HSS control for this MIMO single-tone disturbance rejection problem.

Let $z$ have entries $z=\left[z_{1} z_{2}\right]^{\mathrm{T}}$, so that $z_{1}$ represents the output from microphone 1 and $z_{2}$ represents the output from microphone 2. Measurements of $z$ are obtained at times
TABLE II

DiMENSIONS OF THE ACOUSTIC DRUM

\begin{tabular}{cc}
\hline Length of drum & $0.88 \mathrm{~m}$ \\
\hline Cross sectional area of drum & $0.27 \mathrm{~m}^{2}$ \\
\hline Cross sectional area of speaker & $0.015 \mathrm{~m}^{2}$ \\
\hline
\end{tabular}

$t_{k, 1}, \ldots, t_{k, r} \in\left[t_{k}, t_{k+1}\right]$. It then follows from (2.5) and (2.8) that, for all $i=1, \ldots, r$

$$
z\left(t_{k, i}\right)=\left[\begin{array}{l}
z_{\mathrm{s}_{1, k}} \\
z_{\mathrm{s}_{2, k}}
\end{array}\right] \sin \left(\omega t_{k, i}\right)+\left[\begin{array}{l}
z_{\mathrm{c}_{1, k}} \\
z_{\mathrm{c}_{2, k}}
\end{array}\right] \cos \left(\omega t_{k, i}\right)
$$

and, hence, for $i=1,2$

$$
\left[\begin{array}{c}
z_{i}\left(t_{k, 1}\right) \\
\vdots \\
z_{i}\left(t_{k, r}\right)
\end{array}\right]=\left[\begin{array}{cc}
\sin \left(\omega t_{k, 1}\right) & \cos \left(\omega t_{k, 1}\right) \\
\vdots & \vdots \\
\sin \left(\omega t_{k, r}\right) & \cos \left(\omega t_{k, r}\right)
\end{array}\right] \bar{z}_{i, k}
$$

where $\bar{z}_{i, k} \triangleq\left[z_{\mathrm{s}_{i, k}} z_{\mathrm{c}_{i, k}}\right]^{\mathrm{T}}$. A dSPACE 1003 system is used to determine the vector $\bar{z}_{k}=\left[\bar{z}_{1, k}^{\mathrm{T}} \bar{z}_{2, k}^{\mathrm{T}}\right]^{\mathrm{T}}$ from measurements of $z(t)$ by solving the algebraic equation (10.2). The update $\bar{u}_{k}$ is computed by a Simulink implementation of the HSS control algorithm.

To estimate $T$ before system operation, a sinusoidal input with frequency $50 \mathrm{~Hz}$, amplitude $\hat{u}_{1}$ and $\hat{u}_{2}$, and phase angle $\psi_{1}$ and $\psi_{2}$ is applied to the system through actuation speakers 1 and 2, respectively, in $N$ separate trials. The amplitudes $\hat{u}_{1,1}, \ldots, \hat{u}_{1, N}$ and $\hat{u}_{2,1}, \ldots, \hat{u}_{2, N}$, and the phase angles $\psi_{1,1}, \ldots, \psi_{1, N}$ and $\psi_{2,1}, \ldots, \psi_{2, N}$ are chosen randomly for each trial. For all $i=1, \ldots, N$, define $u_{\mathrm{s}_{1, i}}, u_{\mathrm{c}_{1, i}}, u_{\mathrm{s}_{2}, i}$, and $u_{\mathrm{c}_{2, i}}$ by

$$
\begin{aligned}
& u_{\mathrm{s}_{1, i}} \triangleq-\hat{u}_{1, i} \sin \left(\psi_{1, i}\right), u_{\mathrm{c}_{1, i}} \triangleq \hat{u}_{1, i} \cos \left(\psi_{1, i}\right) \\
& u_{\mathrm{s}_{2, i}} \triangleq-\hat{u}_{2, i} \sin \left(\psi_{2, i}\right), u_{\mathrm{c}_{2, i}} \triangleq \hat{u}_{2, i} \cos \left(\psi_{2, i}\right)
\end{aligned}
$$

and define $\bar{u}_{i}$ by $\bar{u}_{i} \triangleq\left[\begin{array}{llll}u_{\mathrm{s}_{1, i}} & u_{\mathrm{c}_{1, i}} & u_{\mathrm{s}_{2, i}} & u_{\mathrm{c}_{2, i}}\end{array}\right]^{\mathrm{T}}$. Let $\bar{z}_{i}$ be the output vector defined in (2.12) corresponding to the input in the $i$ th trial. Next, define $\Delta \hat{U}$ and $\Delta \hat{Z}$ by

$$
\begin{aligned}
& \Delta \hat{U} \triangleq\left[\begin{array}{lll}
\bar{u}_{1}-\bar{u}_{2} & \cdots & \bar{u}_{N-1}-\bar{u}_{N}
\end{array}\right] \\
& \Delta \hat{Z} \triangleq\left[\begin{array}{lll}
\bar{z}_{1}-\bar{z}_{2} & \cdots & \bar{z}_{N-1}-\bar{z}_{N}
\end{array}\right] .
\end{aligned}
$$

The least squares estimate $T_{\mathrm{LS}}$ of $T$ is given by

$$
T_{\mathrm{LS}}=\Delta \hat{Z} \Delta \hat{U}^{\mathrm{T}}\left(\Delta \hat{U} \Delta \hat{U}^{\mathrm{T}}\right)^{-1}
$$

and used in the HSS algorithm.

Figs. 9 and 10 show the performance of HSS control when microphone 1 is at location $\mathrm{A}$, microphone 2 is at location $\mathrm{B}$, and $T=T_{\mathrm{LS}}$. Next, we modify the experiment so that $T_{\mathrm{LS}}$ is no longer a useful estimate, that is, $T$ is uncertain. To do this, at $t \approx 3 \mathrm{~s}$, microphone 2 is moved from location $\mathrm{A}$ to location $\mathrm{C}$, resulting in a change in the system dynamics. Since 


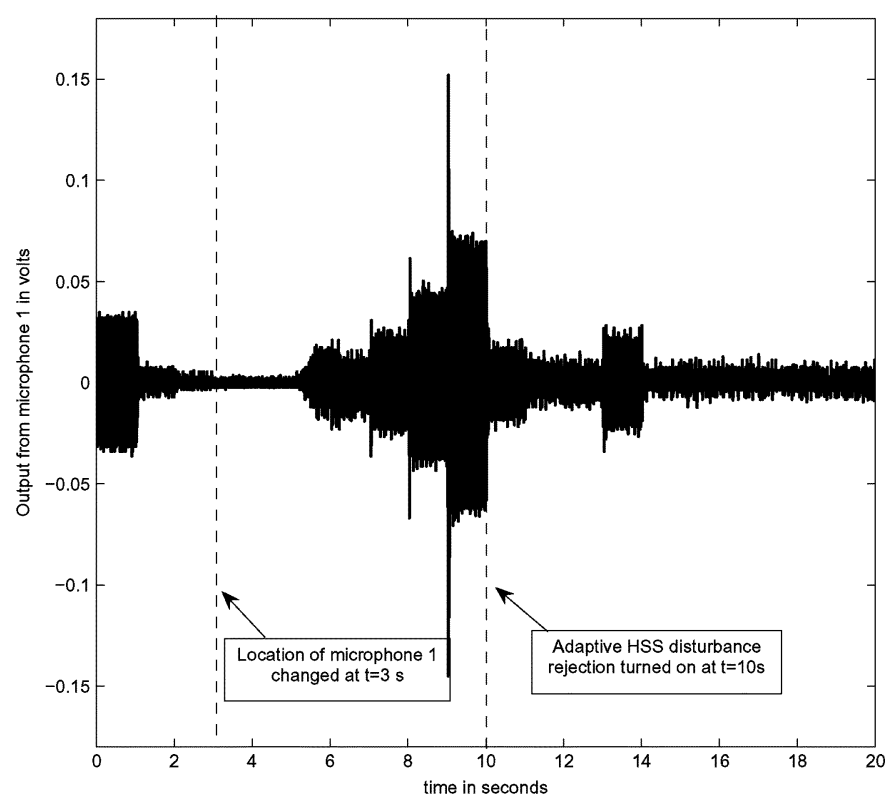

Fig. 9. Experimental disturbance rejection at microphone 1 using nonadaptive and adaptive HSS control. Microphone 1 is moved from Location A to Location $\mathrm{C}$ at $t \approx 3 \mathrm{~s}$ and the output diverges. Adaptive HSS control begins at $t \approx 10 \mathrm{~s}$, and convergence is achieved. Since the control (7.9) is used, the sudden change in the amplitude of the control input at switching instants causes huge transients.

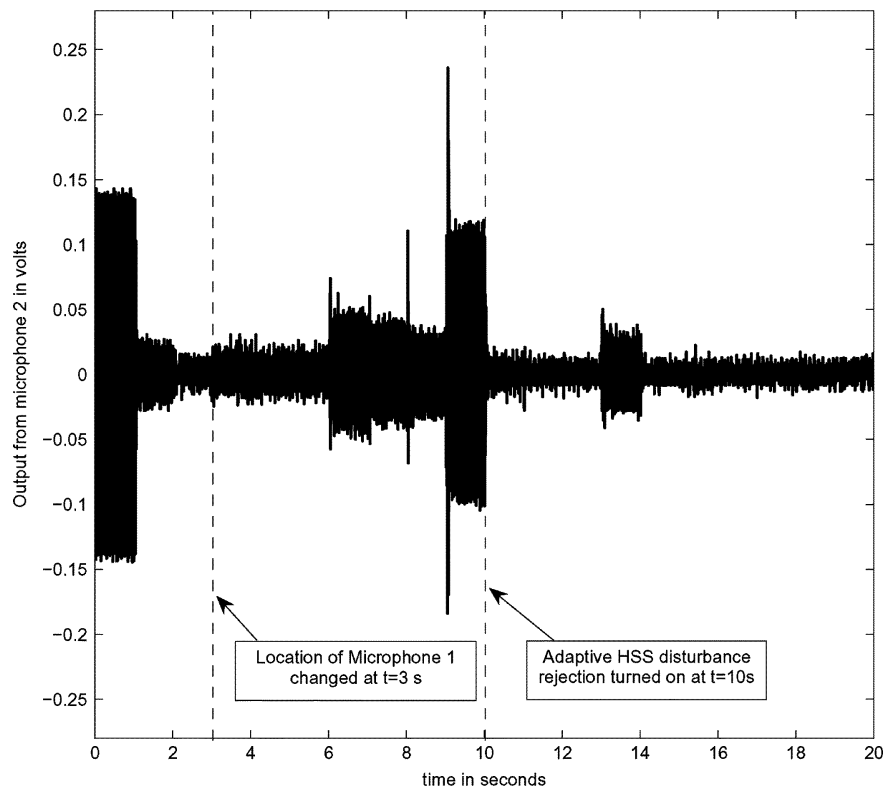

Fig. 10. Experimental output of microphone 2 when nonadaptive and adaptive HSS control are used for disturbance rejection. Microphone 1 is moved from location A to location $\mathrm{C}$ at $t \approx 3 \mathrm{~s}$ and the output of microphone 2 also diverges. Adaptive HSS control begins at $t \approx 10 \mathrm{~s}$, and convergence is achieved. The location of microphone 2 is fixed during the entire experiment. The transient performance is poor due to the sudden change in the amplitude of the control input.

conventional HSS control is unaware of this change, the modified closed-loop system is unstable and the output diverges. At $t \approx 10 \mathrm{~s}$, adaptive HSS control begins, and stability is recovered providing disturbance rejection at the new location. The location of microphone 2 is unaltered for the entire duration of the experiment. Furthermore, the amplitude of the control input $u(t)$ is determined by $\bar{u}_{k}$, which is updated every $1 \mathrm{~s}$ by using (7.9). Next, we repeat the above experiment but determine $u(t)$

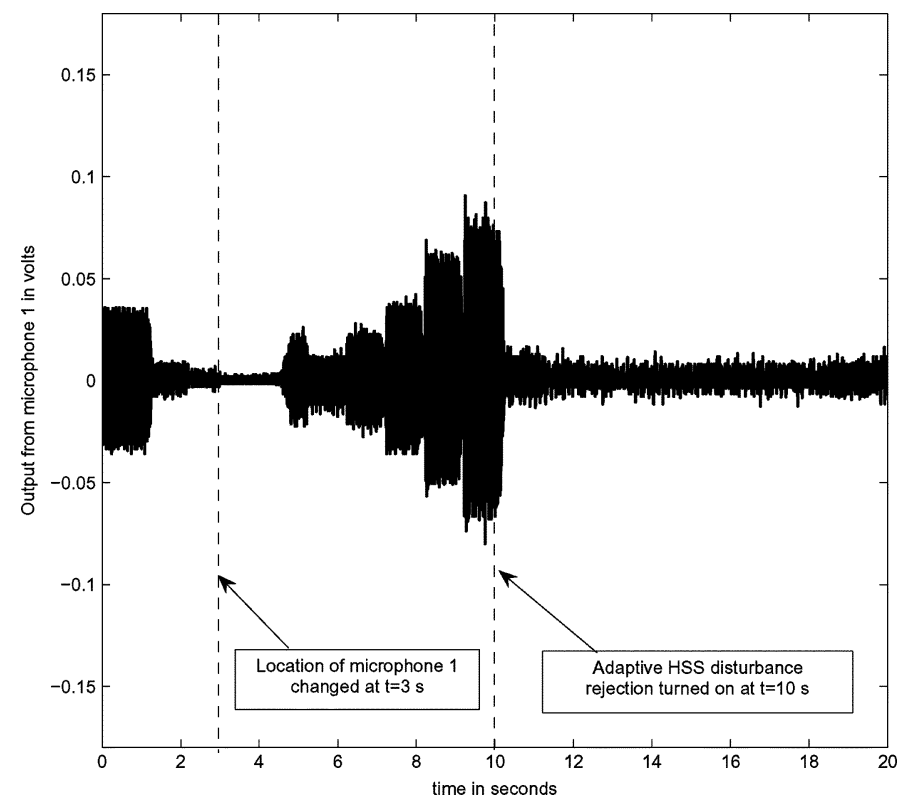

Fig. 11. Experimental disturbance rejection at microphone 1 using nonadaptive and adaptive HSS control. Microphone 1 is moved from location A to location C at $t \approx 3 \mathrm{~s}$ and the output diverges. Adaptive HSS control begins at $t \approx 10 \mathrm{~s}$, and convergence is achieved. By using the interpolating control (9.6), the transient performance improves without any change in the steady-state performance.

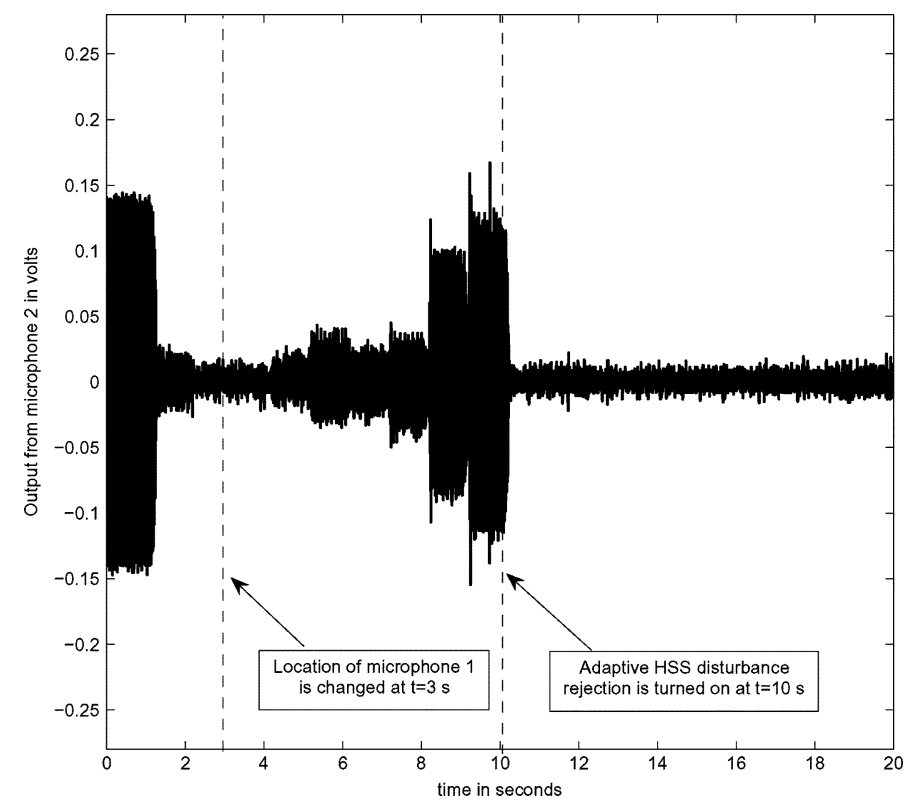

Fig. 12. Experimental output of microphone 2 when nonadaptive and adaptive HSS control are used for disturbance rejection. Microphone 1 is moved from location A to location $\mathrm{C}$ at $t \approx 3 \mathrm{~s}$ and the output of microphone 2 also diverges. Adaptive HSS control begins at $t \approx 10 \mathrm{~s}$, and convergence is achieved. The location of microphone 2 is unaltered during the entire experiment. The interpolating control (9.6) is used.

using the interpolating control (9.6). The output from microphone 1 and microphone 2, when the interpolating control is used, is shown in Figs. 11 and 12. Note that the use of the interpolating control reduces the magnitude of the transients.

Next, we compare the performance of adaptive HSS with and without persistancy. Let $T_{\mathrm{LS}}$ in (10.5) have entries

$$
T_{\mathrm{LS}}=\left[\begin{array}{lll}
T_{\mathrm{LS}_{1}} & \cdots & T_{\mathrm{LS}_{8}}
\end{array}\right]
$$




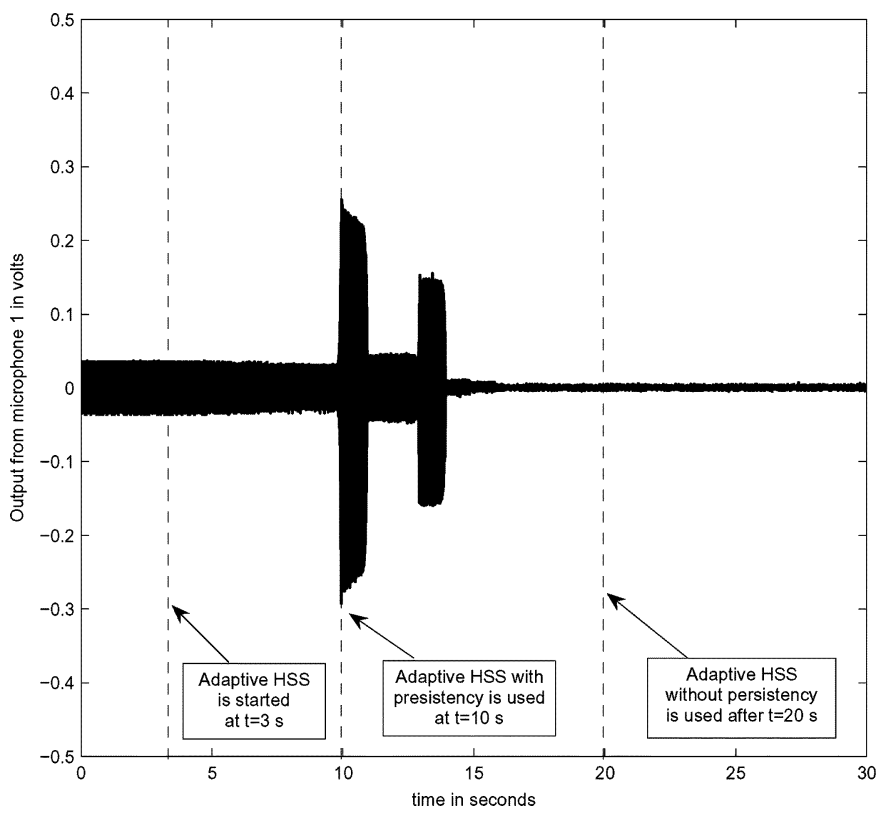

Fig. 13. Experimental output of microphone 1 when nonadaptive HSS control is used with an initial estimate. $\tilde{T}_{\mathrm{LS}}$ of $T$. Although adaptive HSS control begins at $t \approx 3 \mathrm{~s}$, the performance is poor due to the lack of persistent excitation. At $t=10 \mathrm{~s}$, adaptive HSS with persistent excitation is turned on. At $t=20 \mathrm{~s}$, the performance becomes satisfactory and the persistent excitation is turned off. The locations of the microphones are not altered during this experiment. The interpolating control (9.6) is used.

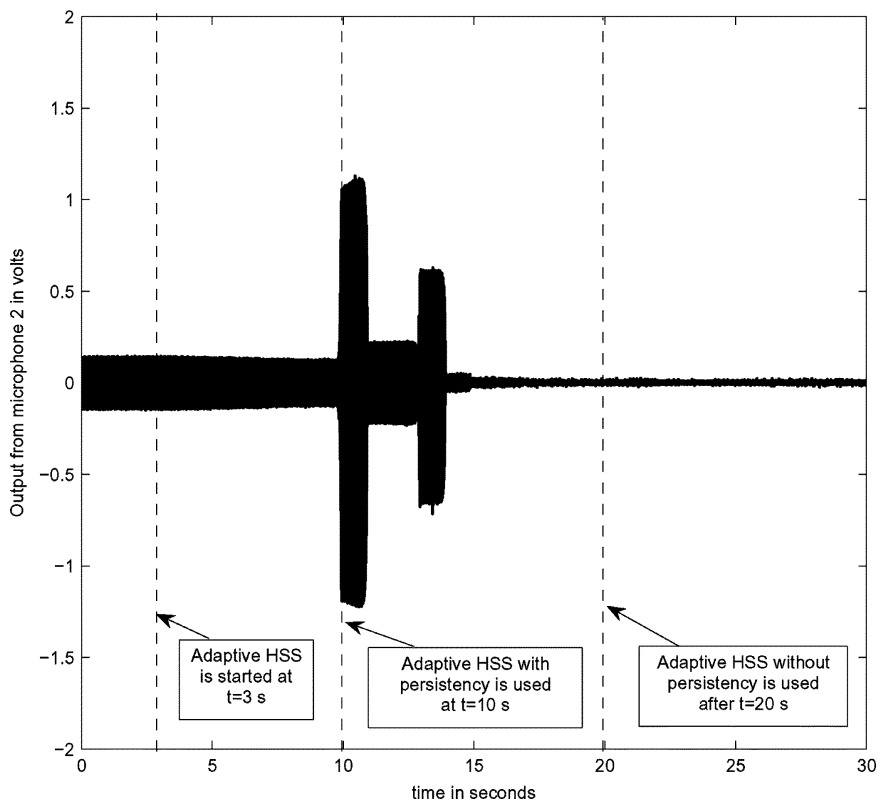

Fig. 14. Experimental output of microphone 2 when adaptive HSS with persistent excitation is used for disturbance rejection.

where, for all $i=1, \ldots, 8, T_{\mathrm{LS}_{i}} \in \mathbb{R}^{8}$. Define, $\tilde{T}_{\mathrm{LS}}$ by

$$
\tilde{T}_{\mathrm{LS}} \triangleq\left[\begin{array}{llll}
T_{\mathrm{LS}_{1}} & 0 & \cdots & 0
\end{array}\right]
$$

Figs. 13 and 14 show the performance of HSS control when microphone 1 and microphone 2 are at location A and location B, respectively, and $\tilde{T}_{\mathrm{LS}}$ is used as an estimate of $T$ in the HSS algorithm. At $t=3 \mathrm{~s}$, adaptive HSS control begins, but since $\Delta u_{k}$ is not persistent, the estimate $\hat{T}_{k}$ does not converge to $T$ and the performance is poor. At $t=10 \mathrm{~s}$, adaptive HSS with persistant is turned on and the experimental results indicate that $\Delta u_{k}$ is persistently exciting. At $t=20 \mathrm{~s}$, the performance is satisfactory and, hence, adaptive HSS without persistency excitation is used after $t=20 \mathrm{~s}$.

\section{CONCLUSION}

In this paper, we developed adaptive HSS control for disturbance rejection. HSS control extends higher harmonic control and convergent control developed for helicopter vibration reduction and rotor imbalance suppression. HSS control is fully MIMO and is applicable to stable systems with tonal or multitonal disturbances. The adaptive HSS algorithm is easy to implement and robust in the sense that no modeling information is required aside from knowledge of the harmonic disturbance spectrum. The implementation of a pseudo-RLS algorithm alleviates the need for offline modeling and, thus, simplifies the implementation of HSS in practice.

\section{APPENDIX \\ HSS CONTROL FOR MULTitone DisturbanCE}

Assume that $w$ is harmonic with frequencies $\omega_{1}, \omega_{2}, \ldots, \omega_{n}$, and that HSS is attained within the time interval $\left[t_{k}, t_{k+1}\right]$. Then $w(t), z(t)$, and $u(t)$ have components

$$
\begin{aligned}
& w(t)=\left[\sum_{i=1}^{n} \hat{w}_{1}^{[i]} \operatorname{Re}\left(e^{\jmath\left(\omega_{i} t+\phi_{1}^{[i]}\right)}\right) \cdots \sum_{i=1}^{n} \hat{w}_{d}^{[i]} \operatorname{Re}\left(e^{\jmath\left(\omega_{i} t+\phi_{d}^{[i]}\right)}\right)\right]^{\mathrm{T}} \\
& z(t)=\left[\sum_{i=1}^{n} \hat{z}_{1, k}^{[i]} \operatorname{Re}\left(e^{\jmath\left(\omega_{i} t+\theta_{1, k}^{[i]}\right)}\right) \cdots \sum_{i=1}^{n} \hat{z}_{p, k}^{[i]} \operatorname{Re}\left(e^{\jmath\left(\omega_{i} t+\theta_{p, k}^{[i]}\right)}\right)\right]^{\mathrm{T}} \\
& u(t)=\left[\sum_{i=1}^{n} \hat{u}_{1, k}^{[i]} \operatorname{Re}\left(e^{\jmath\left(\omega_{i} t+\psi_{1, k}^{[i]}\right)}\right) \cdots \sum_{i=1}^{n} \hat{u}_{m, k}^{[i]} \operatorname{Re}\left(e^{\jmath\left(\omega_{i} t+\psi_{m, k}^{[i]}\right)}\right)\right]^{\mathrm{T}}
\end{aligned}
$$

where $\hat{w}_{j}^{[i]}, \hat{z}_{j, k}^{[i]}, \hat{u}_{j, k}^{[i]}$ are the amplitudes, and $\phi_{j}^{[i]}, \theta_{j, k}^{[i]}$, and $\psi_{j, k}^{[i]}$ are the phase angles of the $i$ th harmonic of the $j$ th component of $w(t), z(t)$, and $u(t)$, respectively. Define $w_{\mathbf{s}_{j}}^{[i]}, w_{\mathbf{c}_{j}}^{[i]}, z_{\mathbf{s}_{j, k}}^{[i]}, z_{\mathbf{c}_{j, k}}^{[i]}$, $u_{\mathbf{s}_{j, k}}^{[i]}$, and $u_{\mathrm{c}_{j, k}}^{[i]}$ by

$$
\begin{array}{cc}
w_{\mathrm{s}_{j}}^{[i]} \triangleq-\hat{w}_{j}^{[i]} \sin \left(\phi_{j}^{[i]}\right), & w_{\mathrm{c}_{j}} \triangleq \hat{w}_{j}^{[i]} \cos \left(\phi_{j}^{[i]}\right) \\
z_{\mathrm{s}_{j, k}}^{[i]} \triangleq-\hat{z}_{j, k}^{[i]} \sin \left(\theta_{j, k}^{[i]}\right), & z_{\mathrm{c}_{j, k}}^{[i]} \triangleq \hat{z}_{j, k}^{[i]} \cos \left(\theta_{j, k}^{[i]}\right) \\
u_{\mathrm{s}_{j, k}}^{[i]} \triangleq-\hat{u}_{j, k}^{[i]} \sin \left(\psi_{j, k}^{[i]}\right), & u_{\mathrm{c}_{j, k}}^{[i]} \triangleq \hat{u}_{j, k}^{[i]} \cos \left(\psi_{j, k}^{[i]}\right) .
\end{array}
$$

Define $w^{[i]} \in \mathbb{R}^{2 d}, z_{k}^{[i]} \in \mathbb{R}^{2 p}$, and $u_{k}^{[i]} \in \mathbb{R}^{2 m}$ by

$$
\begin{aligned}
w^{[i]} & \triangleq\left[w_{\mathbf{s}_{1}}^{[i]} w_{\mathbf{c}_{1}}^{[i]} \cdots w_{\mathbf{s}_{d}}^{[i]} w_{\mathbf{c}_{d}}^{[i]}\right]^{\mathrm{T}} \\
z_{k}^{[i]} & \triangleq\left[z_{\mathbf{s}_{1, k}}^{[i]} z_{\mathbf{s}_{1, k}}^{[i]} \cdots z_{\mathbf{s}_{p, k}}^{[i]} z_{\mathbf{c}_{p, k}}^{[i]}\right]^{\mathrm{T}} \\
u_{k}^{[i]} & \triangleq\left[u_{\mathbf{s}_{1, k}}^{[i]} u_{\mathbf{c}_{1, k}}^{[i]} \cdots u_{\mathbf{s}_{m, k}}^{[i]} u_{\mathbf{c}_{m, k}}^{[i]}\right]^{\mathrm{T}} .
\end{aligned}
$$


Define $\bar{w} \in \mathbb{R}^{2 d n}, \bar{z}_{k} \in \mathbb{R}^{2 p n}$, and $\bar{u}_{k} \in \mathbb{R}^{2 m n}$ by

$$
\begin{aligned}
& \bar{w} \triangleq\left[\left(w^{[1]}\right)^{\mathrm{T}} \cdots\left(w^{[n]}\right)^{\mathrm{T}}\right]^{\mathrm{T}}, \quad \bar{z}_{k} \triangleq\left[\left(z_{k}^{[1]}\right)^{\mathrm{T}} \cdots\left(z_{k}^{[n]}\right)^{\mathrm{T}}\right]^{\mathrm{T}} \\
& \bar{u}_{k} \triangleq\left[\left(u_{k}^{[1]}\right)^{\mathrm{T}} \cdots\left(u_{k}^{[n]}\right)^{\mathrm{T}}\right]^{\mathrm{T}} .
\end{aligned}
$$

The system dynamics can now be represented by (2.11), with $T \in \mathbb{R}^{2 p n \times 2 m n}$ and $W \in \mathbb{R}^{2 p n \times 2 d n}$ defined by

$$
\begin{array}{r}
T \triangleq\left[\begin{array}{cccc}
T_{\omega_{1}} & 0_{2 p \times 2 m} & \cdots & 0_{2 p \times 2 m} \\
0_{2 p \times 2 m} & T_{\omega_{2}} & 0_{2 p \times 2 m} & \vdots \\
\vdots & \ddots & \ddots & \vdots \\
0_{2 p \times 2 m} & \ldots & \ldots & T_{\omega_{n}}
\end{array}\right] \\
W \triangleq\left[\begin{array}{cccc}
W_{\omega_{1}} & 0_{2 p \times 2 d} & \cdots & 0_{2 p \times 2 d} \\
0_{2 p \times 2 d} & W_{\omega_{2}} & 0_{2 p \times 2 d} & \vdots \\
\vdots & \ddots & \ddots & \vdots \\
0_{2 p \times 2 d} & \ldots & \ldots & W_{\omega_{n}}
\end{array}\right]
\end{array}
$$

where $T_{\omega_{i}} \in \mathbb{R}^{2 p \times 2 m}$ and $W_{\omega_{i}} \in \mathbb{R}^{2 p \times 2 d}$ are defined by (2.14), with $\omega_{1}$ replaced by $\omega_{i}$.

\section{REFERENCES}

[1] J. Hong and D. S. Bernstein, "Bode integral constraints, colocation, and spillover in active noise and vibration control," IEEE Trans. Contr. Syst. Technol., vol. 6, no. 1, pp. 111-120, Jan. 1998.

[2] W. Messner and M. Bodson, "Design of adaptive feedforward algorithms using internal model equivalence," Int. J. Adapt. Contr. Signal Process., vol. 9, no. 2, pp. 199-212, Mar. 1995.

[3] M. Bodson, A. Sacks, and P. Khosla, "Harmonic generation in adaptive feedforward cancellation schemes," IEEE Trans. Autom. Contr., vol. 39, no. 9, pp. 1939-1943, Sep. 1994.

[4] S. M. Kuo and D. R. Morgan, Active Noise Control Systems. New York: Wiley-Interscience, 1996.

[5] J. E. Greenberg, "Modified LMS algorithms for speech processing with an adaptive noise canceller," IEEE Trans. Speech Audio Process., vol. 6, no. 4, pp. 338-351, Jul. 1998.

[6] S. J. Elliott and P. J. Nelson, "Active noise control," IEEE Signal Process. Mag., vol. 10, no. 4, pp. 12-35, Oct. 1993.

[7] Y. Hinamoto and H. Sakai, "Analysis of the filtered-X LMS algorithm and a related new algorithm for active control of multitonal noise," IEEE Trans. Speech Audio Process., vol. 14, no. 1, pp. 123-130, Jan. 2006.

[8] E. Bjarnason, "Analysis of the filtered-X algorithm," IEEE Trans. Speech Audio Process., vol. 3, no. 6, pp. 504-514, Nov. 1995.

[9] J. Tapia and S. M. Kuo, "New adaptive online modeling technique for active noise control systems," in Proc. IEEE Conf. Syst. Eng., 1990, pp. 280-283.

[10] M. Rupp and A. H. Sayed, "Robust FxLMS algorithms with improved convergence performance," IEEE Trans. Speech Audio Process., vol. 6, no. 1, pp. 78-85, Jan. 1998.

[11] M. Bodson and S. C. Scott, "Adaptive algorithms for the rejection of sinusoidal disturbances with unknown frequency," Automatica, vol. 33, no. 12, pp. 2213-2221, Dec. 1997.

[12] M. Bodson, "Performance of an adaptive algorithm for sinusoidal disturbance rejection in high noise," Automatica, vol. 37, no. 7, pp. 1133-1140, Jul. 2001.

[13] R. Venugopal and D. S. Bernstein, "Adaptive disturbance rejection using ARMARKOV/toeplitz models," IEEE Trans. Contr. Syst. Technol., vol. 8, no. 2, pp. 257-269, Mar. 2000.

[14] J. T. Pearson and R. M. Goodall, "Adaptive schemes for the active control of helicopter structural response," IEEE Trans. Contr. Syst. Technol., vol. 2, no. 2, pp. 61-72, Jun. 1994.
[15] P. P. Friedmann and T. A. Millott, "Vibration reduction in rotorcraft using active control: A comparison of various approaches," J. Guid. Contr. Dyn., vol. 18, no. 4, pp. 664-673, 1995.

[16] M. Lovera, P. Colaneri, C. Malpicia, and R. Celi, "Closed-loop aeromechanical stability analysis of HHC and IBC, with application to a hingeless rotor helicopter," in Proc. 29th Eur. Rot. Forum, 2003, pp. 43.1-43.14.

[17] K. P. Nygren and D. P. Schrage, "Fixed-gain versus adaptive higher harmonic control simulation," J. Amer. Helicopter Soc., vol. 34, no. 3, pp. 51-58, Jul. 1989.

[18] J. A. Molusis, C. E. Hammond, and J. H. Cline, "A unified approach to the optimal design of adaptive and gain scheduled controllers to achieve minimum helicopter rotor vibration," J. Amer. Helicopter Soc., vol. 28, no. 2, pp. 9-18, Apr. 1983.

[19] C. R. Knospe, R. W. Hope, S. M. Tamer, and S. J. Fedigan, "Robustness of adaptive unbalance control of rotors with magnetic bearings," J. Vibr. Contr., vol. 2, no. 2, pp. 33-52, Feb. 1996.

[20] S. R. Hall and N. M. Werely, "Performance of higher harmonic control algorithms for helicopter vibration reduction," J. Guid. Contr. Dyn., vol. 16, no. 4, pp. 793-797, 1993.

[21] W. Johnson, Ames Research Center, Moffett Field, CA, "Self-tuning regulators for multicyclic control of helicopter vibration," NASA Tech. Paper, 1982.

[22] K. J. Astrom and B. Wittenmark, Adaptive Control. Reading, MA: Addison-Wesley, 1995.

[23] C. R. Knospe, R. W. Hope, S. J. Fedigan, and R. D. Williams, "Experiments in the control of unbalance response using magnetic bearings," IEEE/ASME Trans. Mechatronics, vol. 5, no. 4, pp. 385-400, Jun. 1995.

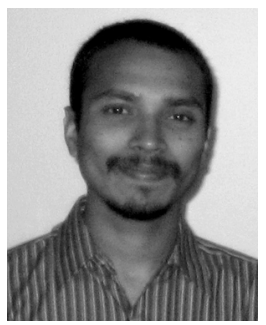

Jaganath Chandrasekar recieved the B.E. (Hons) degree in mechanical engineering from Birla Institute of Technology and Science, Pilani, India, and the M.S. degree in aerospace engineering from the University of Michigan, Ann Arbor, in 2002 and 2004, respectively. He is currently pursuing the Ph.D. degree in aerospace engineering at the same university.

His research interests include active noise control, control of robotic manipulators, and data assimilation of high-dimensional nonlinear systems.

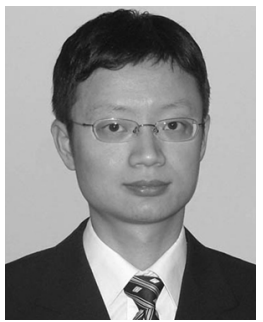

Li Liu received the B.E. degree from Beijing University of Aeronautics and Astronautics, Beijing, China, and the M.S.E. and Ph.D. degrees from the University of Michigan, Ann Arbor, in 1997 and 2005, respectively, all in aerospace engineering.

He is currently a Postdoctoral Research Fellow in the Department of Aerospace Engineering at the University of Michigan. His main research interests are rotorcraft aeroelasticity, aeroacoustics, and active vibration and noise reduction in helicopters.

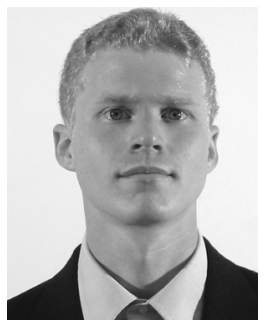

Dan Patt received the B.S.E., M.S.E., and Ph.D. degrees in aerospace engineering from the University of Michigan, Ann Arbor, in 2000, 2002, and 2004, respectively.

$\mathrm{He}$ is currently employed as a Research Scientist and Engineer in the Advanced Concept Exploration Division of Boeing Phantom Works, Irvine, CA. His current research interests include unmanned aerial vehicles, vehicle autonomy, rotorcraft aeroelasticity, and structural dynamics. 


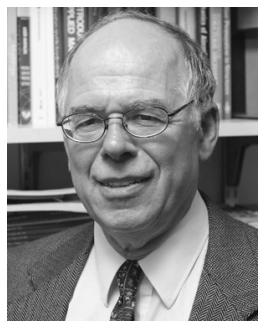

Peretz P. Friedmann received the Sc.D. degree in aeronautics and astronautics from the Massachusetts Institute of Technology, Cambridge, in 1972.

He has been with the University of Michigan, Ann Arbor, since January 1999, where he has been the Francois-Xavier Bagnoud Professor of Aerospace Engineering and Director of the FXB Center for Rotary and Fixed Wing Air Vehicle Design. Between 1972 and 1998, he was a Professor in the Mechanical and Aerospace Engineering Department at the University of California at Los Angeles, Los Angeles. He has been engaged in research on rotary and fixed wing aeroelasticity, structural dynamics, unsteady aerodynamics, and numerical methods. His recent research has focused on vibration and noise reduction in helicopters using actively controlled flaps and aerothermoelasticity of hypersonic vehicles.

Dr. Friedmann was a recipient of the ASME Structures and Materials Award in 1984, the AIAA Structures, Structural Dynamics, and Materials Award in 1996, the Spirit of St. Louis Medal, and the ASME/Boeing Structures and Materials Award in 2004. He is a member of the ASME (2003), and Fellow of the AHS (2004) and AIAA (1991).

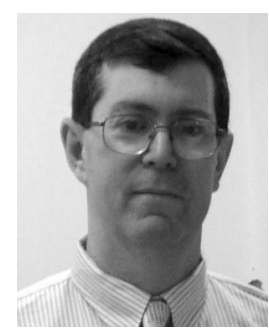

Dennis S. Bernstein received the Sc.B. degree in applied mathematics from Brown University, Providence, RI, in 1977, and the Ph.D. degree in control engineering from the University of Michigan, Ann Arbor, in 1982.

$\mathrm{He}$ is currently a Professor in the Aerospace Engineering Department at the University of Michigan. His interests encompass all aspects of control for aerospace applications, with an emphasis on identification and adaptive control for vibration and flow control. He is the author of Matrix Mathematics: Theory, Facts, and Formulas with Application to Linear Systems Theory (Princeton Univ. Press, 2004) 